\title{
Performance of the modulation diversity technique for $\kappa-\mu$ fading channels
}

\author{
Rafael F Lopes ${ }^{1,3,4^{*}}$, Wamberto J L Queiroz ${ }^{2,3}$, Waslon T A Lopes 2,3 and Marcelo S Alencar ${ }^{2,3}$
}

\begin{abstract}
The performance of wireless communication systems can be significantly improved using the modulation diversity technique, which is based on the combination of a suitable choice of the reference angle of a signal constellation with independent interleaving of the symbol components. This technique has been evaluated considering different fading channel models, such as Rayleigh, Rice and Nakagami-m. However, in some specific scenarios, the tails of those fading distributions do not properly fit the experimental measured data, which demands the use of more general channel distributions. This article presents a performance evaluation of the modulation diversity technique for $\kappa-\mu$ fading channels. New expressions for the PEP (Pairwise Error Probability) are obtained using numerical integration, series representation and upper/lower bounds. The evaluation, based on Monte Carlo simulation, demonstrates that the performance gain of the modulation diversity increases as the fading becomes more severe. Communications channels exhibit some degree of time correlation, which cannot be perfectly estimated, affecting the performance of the modulation diversity system. Thus, a performance evaluation of the system, concerning the presence of temporal correlation and estimation errors in the channel is also presented in the article.
\end{abstract}

Keywords: Modulation diversity, $\kappa-\mu$ fading, Wireless communications

\section{Introduction}

Multipath fading can significantly degrade the performance of communication systems. Several techniques have been proposed to mitigate the effects of fading to improve their performance, and diversity techniques appear as a solution to the problem [1-3]. Diversity techniques provide replicas of the transmitted signals to the receiver [4].

A useful diversity technique is based on the combination of a suitable choice of a reference constellation rotation angle $(\theta)$ with the independent interleaving of the symbol components before the transmission [4,5]. The optimal rotation angle depends on the chosen constellation order $(M)$, as well as on the fading severity degree [6]. In this article, this technique is referred to as modulation diversity $[4,7]$, but it is also known as constellation rotation [8], signal space diversity $[9,10]$ and rotation and component interleaving diversity [11].

*Correspondence: rafael.fernandes@ee.ufcg.edu.br

${ }^{1}$ D.Sc. Student of the Federal University of Campina Grande (UFCG), Electrical Engineering Post Graduate Program-COPELE, Campina Grande, Brazil

3 Institute for Advanced Studies in Communications, Campina Grande, Brazil Full list of author information is available at the end of the article
The performance of the modulation diversity technique has been evaluated considering different scenarios, which include $M$-ary phase shift keying ( $M$-PSK) and $M$-ary quadrature amplitude modulation ( $M$-QAM) constellations for Rayleigh fading channels [5,7,8,12], Rician fading channels $[13,14]$ and Nakagami- $m$ fading channels $[11,15]$. However, in some specific scenarios, the tails of those fading distributions do not properly fit the experimental measured data, as discussed in [16].

Yacoub [17] proposed two fading distributions, namely $\kappa-\mu$ and $\eta-\mu$, to allow flexibility to model the wireless channels fading fluctuations. Those distributions are fully characterized in terms of measurable physical parameters. The $\kappa-\mu$ distribution includes the Rice (Nakagami- $n$ ), Nakagami- $m$, Rayleigh and One-Sided Gaussian distributions as special cases. On the other hand, the $\eta-\mu$ distribution includes the Hoyt (Nakagami- $q$ ), Nakagami- $m$, Rayleigh and One-Sided Gaussian distributions as special cases. As discussed in [17], the versatility provided by the use of those distributions shows a good fit to experimental data (particularly for low values of the fading envelope) [17]. It is worth to mention that this article is focused on

\section{是 Springer}

(c) 2013 Lopes et al: licensee Springer. This is an Open Access article distributed under the terms of the Creative Commons Attribution License (http://creativecommons.org/licenses/by/2.0), which permits unrestricted use, distribution, and reproduction in any medium, provided the original work is properly cited. 
$\kappa-\mu$ distribution, but the proposed methodology could be extended to $\eta-\mu$ distribution.

Recently, many articles deal with the $\kappa-\mu$ and $\eta-\mu$ distributions. Considering the application of diversity techniques, useful formulas for the pdf (probability density function) and CDF (Cumulative Distribution Function) of the sum of squared $\kappa-\mu$ variates were presented [18], and an analytical expression for the switching rate of a dual branch selection diversity combiner was derived in [19]. A systematic investigation on the fading characteristics experienced in body to body communications channels, for fire and rescue personnel, was presented in [20], and the parameters $\kappa$ and $\mu$ were obtained for transmission in the $2.45 \mathrm{GHz}$ range. Using a similar approach, the authors in reference [21] investigated the distribution of signal phase in body area networks.

This article presents a performance evaluation of the modulation diversity technique for the $\kappa-\mu$ fading distribution. Novel approximate expressions for the PEP (Pairwise Error Probability) for $\kappa-\mu$ fading channels are derived. Based on the degrees of freedom provided by the $\kappa-\mu$ distribution, the optimum rotation angle and the system performance are evaluated for different scenarios, including time correlated channels, subject to estimation errors. It is shown that modulation diversity improves the performance of digital communication systems, especially under severe fading.

The remaining sections are organized as follows. Section 2 presents the system and channel models adopted in this article. An analytical framework for the optimization of modulation diversity technique for $\kappa-\mu$ fading channels is described in Section 3. The performance evaluation results are presented in Section 4. Section 5 discuss the effects of the temporal correlation in the performance of the system, while the results concerning the presence of channel estimation errors are presented in Section 6. Section 7 is devoted to the conclusions.

\section{System and channel models}

The modulation diversity technique mitigates the effects of the multipath fading in the transmitted signals. In this technique, the introduction of redundancy can be obtained by combining the rotation of the signal constellation, by an appropriate reference angle $\theta$, with the independent interleaving of the symbol components. Figure 1 illustrates the block diagram for the modulation diversity technique [4].

The channel model is characterized by a slowly varying flat fading. Thus, the received signal, denoted by $r(t)$, can be written as

$$
\boldsymbol{r}(t)=\boldsymbol{\alpha}(t) e^{j \boldsymbol{\phi}(t)} \boldsymbol{s}(t)+\boldsymbol{n}(t)
$$

in which $\boldsymbol{s}(t)$ represents the transmitted signal, $\boldsymbol{\alpha}(t)$ is the fading amplitude, $\phi(t)$ is the phase shift produced by the channel and $\boldsymbol{n}(t)$ represents the additive noise, modeled as a complex white Gaussian process (AWGN), with zero mean and variance $N_{0} / 2$ by dimension.

The fading amplitude $\boldsymbol{\alpha}(t)$ is modeled as a $\kappa-\mu$ stationary random variable. The $\kappa-\mu$ distribution is a general fading distribution that can be used to represent the small-scale variation of the fading signal in a line-of-sight condition. It is modeled by the parameters $\kappa$ and $\mu$, that define the shape of the distribution. The $\kappa-\mu$ distribution includes Rice $(\kappa=K, \mu=1)$, Nakagami-m $(\kappa \rightarrow 0$, $\mu=m)$, Rayleigh $(\kappa \rightarrow 0, \mu=1)$ and One-Sided Gaussian $(\kappa \rightarrow 0, \mu=1 / 2)$ distributions as special cases [17].

The fading model for the $\kappa-\mu$ distribution considers a signal composed of clusters of multipath waves, propagating in a non-homogeneous environment. The phases of the scattered waves, within each cluster, are random and have similar large delay times. Furthermore, the clusters of multipath waves are assumed to have scattered waves with identical powers, but a dominant component is found within each cluster, which presents an arbitrary power [17].

The $\kappa-\mu$ normalized probability density function (pdf) is expressed as [17]

$$
\begin{aligned}
p(\alpha)= & \frac{2 \mu(1+\kappa)^{\frac{\mu+1}{2}}}{\kappa^{\frac{\mu-1}{2}} \exp [\mu \kappa]} \alpha^{\mu} \exp \left[-\mu(1+\kappa) \alpha^{2}\right] \\
& \times I_{\mu-1}[2 \mu \sqrt{\kappa(1+\kappa)} \alpha], \alpha \geq 0,
\end{aligned}
$$

in which $E\left[\alpha^{2}\right]=1, I_{v}(\cdot)$ denotes the modified Bessel function of the first kind and order $v$ ([22], 8.431), $\kappa \geq 0$ is the ratio between the total power of the dominant components and the total power of the scattered waves, and $\mu>0$ is given by $\mu=\frac{1}{\operatorname{Var}\left[\alpha^{2}\right]} \frac{1+2 \kappa}{(1+\kappa)^{2}}$. It is assumed that the fading amplitude is perfectly estimated at the receiver, i.e., $\hat{\alpha}(t)=\alpha(t)$. Moreover, by coherent detection, the effect of the fading on the phase of the received signal is completely compensated.

For the rotated and interleaved system, the transmitted waveform model can be written as

$$
\begin{aligned}
s(t)= & \sum_{n=-\infty}^{+\infty} x_{n} p\left(t-n T_{S}\right) \cos \left(\omega_{c} t\right) \\
& +\sum_{n=-\infty}^{+\infty} y_{n-k} p\left(t-n T_{S}\right) \sin \left(\omega_{c} t\right)
\end{aligned}
$$

in which $k$ is an integer that represents the delay (expressed in number of symbols) introduced by the interleaving between the I (in-phase) and Q (quadrature) components (also named interleaving depth), $p(t)$ denotes the 


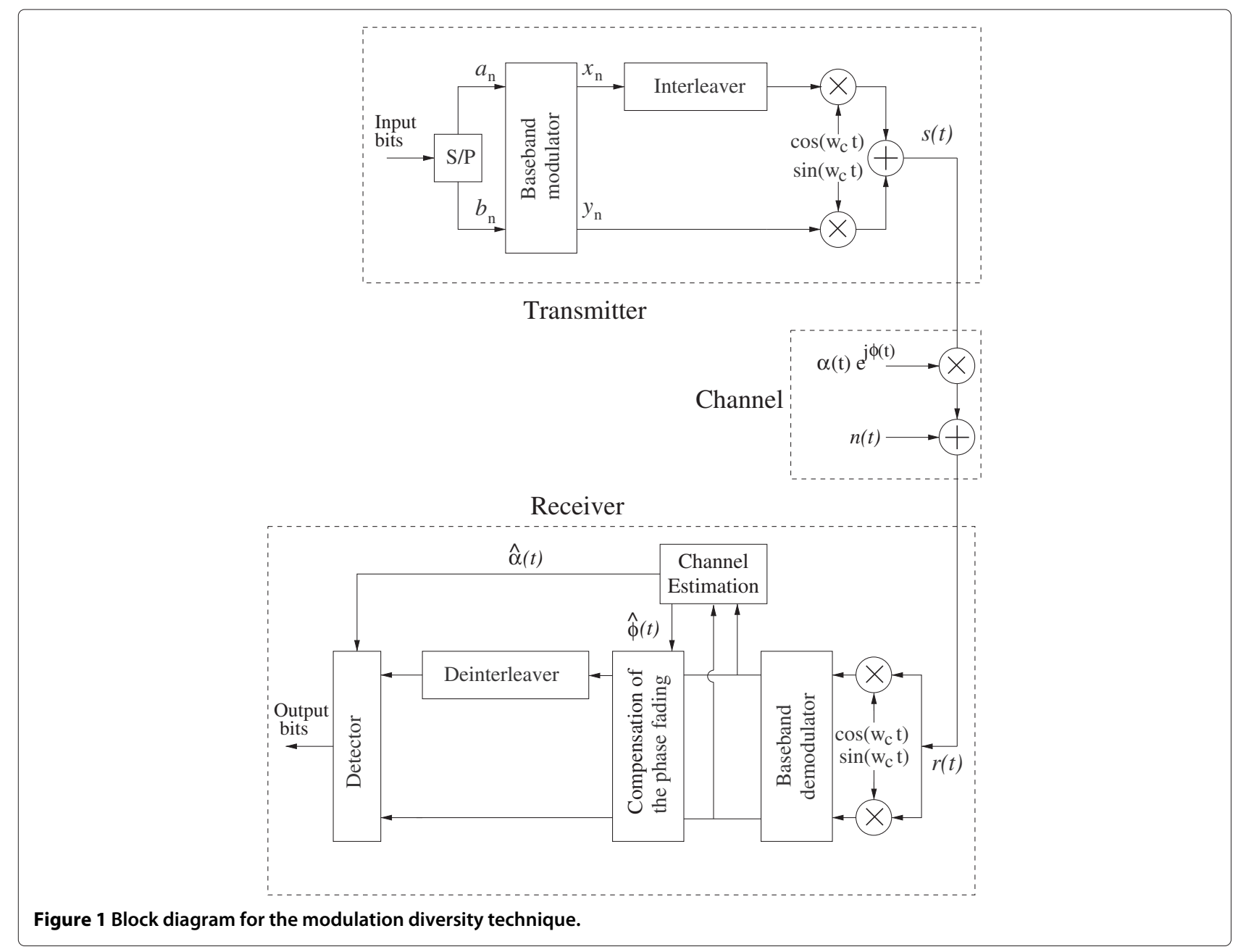

symbol pulse shape, $T_{S}$ is the symbol period, $\omega_{c}$ is the carrier frequency and

$$
\begin{aligned}
& x_{n}=a_{n} \cos \theta-b_{n} \sin \theta, \\
& y_{n}=a_{n} \sin \theta+b_{n} \cos \theta .
\end{aligned}
$$

In addition,

$$
a_{n}, b_{n}= \pm d, \pm 3 d, \ldots, \pm(\sqrt{M}-1) d,
$$

in which $d$ is the minimum distance between the constellation points and $M$ is the modulation order.

Since the transmitted symbols do not share common components, the $I$ and $Q$ components are independently affected by fading. Thus, after the deinterleaving, each received symbol vector $\boldsymbol{r}$ can be expressed as

$$
\begin{aligned}
& \boldsymbol{r}=\left[r_{I}(t), r_{Q}(t)\right], \\
& \boldsymbol{r}=\left[\alpha_{I}(t) s_{I}(t)+n_{I}(t), \alpha_{Q}(t+k) s_{Q}(t)+n_{Q}(t+k)\right],
\end{aligned}
$$

in which $s_{I}(t), s_{Q}(t)$ are the I and Q signal components of the symbol $s(t), \alpha_{I}(t), \alpha_{Q}(t)$ represent the fading that affects the I and Q components, and $n_{I}(t), n_{Q}(t)$ are the $\mathrm{I}$ and $\mathrm{Q}$ components of noise. Finally, the receiver applies maximum likelihood (ML) metric on the deinterleaved signals to detect the source symbol, as follows

$$
\begin{aligned}
& \hat{s}=\underset{s \in \mathcal{S}}{\operatorname{argmin}}\left(|\boldsymbol{r}-\boldsymbol{\alpha} \odot \boldsymbol{s}|^{2}\right), \\
& \hat{s}=\underset{s \in \mathcal{S}}{\operatorname{argmin}}\left(\left|r_{I}(t)-\alpha_{I}(t) s_{I}\right|^{2}+\left|r_{Q}(t)-\alpha_{Q}(t+k) s_{Q}\right|^{2}\right),
\end{aligned}
$$

in which $|\cdot|$ denotes the standard Euclidean norm, $\odot$ represents the component-wise product and $\mathcal{S}$ represents the signal constellation with $M$ signals.

\section{Optimization analysis of the modulation diversity technique for $\boldsymbol{\kappa}-\boldsymbol{\mu}$ fading channel}

The constellation rotation angle $\theta$ represents an important design criterion of the modulation diversity technique. However, the optimal rotation angle depends on the chosen constellation order $(M)$ and the fading severity degree (defined by the $\kappa$ and $\mu$ parameters). The optimal rotation angle evaluation can be accomplished in 
two ways: (a) by using Monte Carlo simulation; or (b) by the optimization of the system SER (Symbol Error Rate) expression.

The first approach requires more computing power than the second one, since many simulations must be performed in different settings and with different rotation angles. On the other hand, obtaining an exact closed-form expression for the SER of diversity modulation systems is a difficult problem, and a common approach to evaluate the error probability of a two-dimensional signal constellation is the use of upper bounds, such as the union bound (UB) [23]. Assuming equiprobable symbols, the SER is upper bounded by [12]

$$
P_{e} \leq P_{e}^{\mathrm{UB}}=\frac{1}{M} \sum_{s \in \mathcal{S}} \sum_{\substack{\hat{s} \in \mathcal{S} \\ s \neq \hat{s}}} P(s \rightarrow \hat{s}),
$$

in which $\mathcal{S}$ represents the signal constellation with $M$ signals and $P(s \rightarrow \hat{s})$ is the pairwise error probability (PEP) and $\hat{s}$ is estimated by the receiver when $s$ has been transmitted (i.e., if $|\boldsymbol{r}-\boldsymbol{\alpha} \odot \hat{\boldsymbol{s}}|^{2}<|\boldsymbol{r}-\boldsymbol{\alpha} \odot \boldsymbol{s}|^{2}$ ). Furthermore, if only the nearest neighbors of each symbol are considered in the performance evaluation, $P_{e}$ can be approximated by

$$
P_{e} \approx P_{e}^{\mathrm{NN}}=\frac{1}{M} \sum_{s \in \mathcal{S}} \sum_{\hat{s} \in \mathcal{N}(s)} P(s \rightarrow \hat{s}),
$$

in which $\mathcal{N}(s)$ is the set of the nearest neighbors of $s$ in $\mathcal{S}$.

Since interleaving is employed for the transmitted symbols, the I and Q symbol components experience independent fading. Let $\alpha_{I}$ and $\alpha_{Q}$ be the $\kappa-\mu$ distributed fading amplitudes in the I and Q channels, respectively. Thus, the PEP for a system with modulation diversity, subject to $\kappa-\mu$ fading, is given by [23]

$$
\begin{aligned}
P(s \rightarrow \hat{s})= & \int_{0}^{\infty} \int_{0}^{\infty} Q\left(\sqrt{\left.\frac{\bar{\gamma} d_{I}^{2} \alpha_{I}^{2}}{2}+\frac{\bar{\gamma} d_{Q}^{2} \alpha_{Q}^{2}}{2}\right)}\right. \\
& \times p\left(\alpha_{I}\right) p\left(\alpha_{Q}\right) d \alpha_{I} d \alpha_{Q}, \\
P(s \rightarrow \hat{s})= & \frac{4 \mu^{2}(1+\kappa)^{\mu+1}}{\kappa^{\mu-1} \exp [2 \mu \kappa]} \\
& \times \int_{0}^{\infty} \int_{0}^{\infty} Q\left(\sqrt{\left.\frac{\bar{\gamma} d_{I}^{2} \alpha_{I}^{2}}{2}+\frac{\bar{\gamma} d_{Q}^{2} \alpha_{Q}^{2}}{2}\right)}\right. \\
& \times \alpha_{I}^{\mu} \alpha_{Q}^{\mu} \exp \left[-\mu(1+\kappa)\left(\alpha_{I}^{2}+\alpha_{Q}^{2}\right)\right] . \\
I_{\mu-1} & {\left[2 \mu \sqrt{\kappa(1+\kappa)} \alpha_{I}\right] } \\
I_{\mu-1} & {\left[2 \mu \sqrt{\kappa(1+\kappa)} \alpha_{Q}\right] d \alpha_{I} d \alpha_{Q}, }
\end{aligned}
$$

in which $\bar{\gamma}$ is the channel mean SNR, and $d_{I}^{2}, d_{Q}^{2}$ are the Euclidean distances between the symbols $s$ and $\hat{s}$ in the I and Q components, respectively. The distances are given by

$$
\begin{aligned}
& d_{I}^{2}=\left[\cos \left(\phi_{1}+\theta\right)-\cos \left(\phi_{2}+\theta\right)\right]^{2}, \\
& d_{Q}^{2}=\left[\sin \left(\phi_{1}+\theta\right)-\sin \left(\phi_{2}+\theta\right)\right]^{2},
\end{aligned}
$$

in which $\phi_{1}, \phi_{2}$ represent the phases of the two signal constellation points under consideration.

Applying Craig's formula for the $Q(\cdot)$ function [24], given by

$$
Q(x)=\frac{1}{\pi} \int_{0}^{\pi / 2} \exp \left(-\frac{x^{2}}{2 \sin ^{2} \phi}\right) d \phi,
$$

into (12), the PEP expression becomes

$$
\begin{aligned}
& P(s \rightarrow \hat{s})= \frac{4 \mu^{2}(1+\kappa)^{\mu+1}}{\pi \kappa^{\mu-1} \exp [2 \mu \kappa]} \\
& \times \int_{0}^{\pi / 2} \int_{0}^{\infty} \int_{0}^{\infty} \exp \left[-\frac{\bar{\gamma} d_{I}^{2}}{4 \sin ^{2} \phi} \alpha_{I}^{2}\right] \\
& \times \exp \left[-\frac{\bar{\gamma} d_{Q}^{2}}{4 \sin ^{2} \phi} \alpha_{Q}^{2}\right] \alpha_{I}^{\mu} \exp \left[-\mu(1+\kappa) \alpha_{I}^{2}\right] . \\
& I_{\mu-1} {\left[2 \mu \sqrt{\kappa(1+\kappa)} \alpha_{I}\right] \alpha_{Q}^{\mu} \exp \left[-\mu(1+\kappa) \alpha_{Q}^{2}\right] } \\
& I_{\mu-1}\left[2 \mu \sqrt{\kappa(1+\kappa)} \alpha_{Q}\right] d \alpha_{I} d \alpha_{Q} d \phi .
\end{aligned}
$$

The integral in (16) can be calculated in different ways, as presented in the following sections.

\subsection{Exact numerical integration}

Different numerical integration techniques can be used to calculate the PEP. In order to make the calculations easier and computationally efficient, the authors have simplified (16). After performing the integration in $\alpha_{I}$ and $\alpha_{Q}$, using the change of variable $x=\cos ^{2} \phi$ and some analytical manipulations, the PEP expression becomes

$$
P(s \rightarrow \hat{s})=\frac{1}{2 \pi\left[\left(1+c_{I}\right)\left(1+c_{Q}\right)\right]^{\mu}} \cdot \Upsilon\left(\kappa, \mu, c_{I}, c_{Q}\right),
$$

in which $c_{I}=\frac{\bar{\gamma} d_{I}^{2}}{4 \mu(1+\kappa)}, c_{Q}=\frac{\bar{\gamma} d_{Q}^{2}}{4 \mu(1+\kappa)}$ and $\Upsilon(\cdot)$ is a special defined function that should be numerically evaluated. This function is defined as

$$
\begin{aligned}
\Upsilon(a, b, c, d)= & \int_{0}^{1} x^{-1 / 2}(1-x)^{2 b-1 / 2} \\
& \times\left[\left(1-\frac{x}{1+c}\right)\left(1-\frac{x}{1+d}\right)\right]^{-b} \\
& \exp \left[-a b\left(\frac{c}{1+c-x}+\frac{d}{1+d-x}\right)\right] d x
\end{aligned}
$$


The function in (18) can be computed using a software package, such as Mathematica, Maple or Matlab.

\subsection{Series representation}

An alternative form for the PEP can be obtained using a series representation for the Bessel function of the first kind in the integrand of (16), which can be expressed by the following expression ([22], 8.445)

$$
I_{\nu}(x)=\sum_{m=0}^{\infty} \frac{1}{m ! \Gamma(m+v+1)}\left(\frac{x}{2}\right)^{2 m+v} .
$$

Introducing (19) into (16), performing the integrations and other analytical manipulations, the PEP expression becomes

$$
\begin{aligned}
& P(s \rightarrow \hat{s})= \frac{\exp [-2 \mu \kappa]}{2 \pi\left[\left(1+c_{I}\right)\left(1+c_{Q}\right)\right]^{\mu}} \\
& \times \sum_{m=0}^{\infty} \sum_{n=0}^{\infty} \frac{(\mu \kappa)^{m+n} \mathrm{~B}\left(m+n+2 \mu+\frac{1}{2}, \frac{1}{2}\right)}{m ! n !\left(1+c_{I}\right)^{m}\left(1+c_{Q}\right)^{n}} . \\
& F_{1}\left(\frac{1}{2}, m+\mu, n+\mu, m+n+2 \mu+1 ; \frac{1}{1+c_{I}}, \frac{1}{1+c_{Q}}\right),
\end{aligned}
$$

in which $F_{1}(\cdot)$ represents the Appell hypergeometric function ([22], 9.180.1) and $\mathrm{B}(\cdot)$ is the Beta function ([22], 8.380.1).

The infinite series form presented in (20) can be truncated to a few number of terms, becoming an approximation for the PEP expression. Thus, (20) can be rewritten as

$$
\begin{aligned}
P(s \rightarrow \hat{s}) \geq & \frac{\exp [-2 \mu \kappa]}{2 \pi\left[\left(1+c_{I}\right)\left(1+c_{Q}\right)\right]^{\mu}} \\
& \times \sum_{m=0}^{W} \sum_{n=0}^{W} \frac{(\mu \kappa)^{m+n} \mathrm{~B}\left(m+n+2 \mu+\frac{1}{2}, \frac{1}{2}\right)}{m ! n !\left(1+c_{I}\right)^{m}\left(1+c_{Q}\right)^{n}} \\
& \times F_{1}\left(\frac{1}{2}, m+\mu, n+\mu, m+n+2 \mu+1\right. \\
& \left.\frac{1}{1+c_{I}}, \frac{1}{1+c_{Q}}\right) .
\end{aligned}
$$

The number of terms in each series (denoted by $W$ and assumed equal in both summations) should be adjusted according to the severity of the fading. Since the PEP approximation is composed of a double summation, a number of $W^{2}$ terms should be computed. Figure 2 depicts the PEP approximation based on the series representation considering different values of $W$ and the randomly chosen symbols $0.3162+0.9487 j$ and $0.9487-$ $0.3162 j$.

As can be seen in Figure 2a, a small number of terms is required to obtain a precise approximation when the channel is characterized by severe fading $(\kappa=0.5$, $\mu=1.5)-$ in this case, a total number of $W^{2}=16$ terms is used. However, in a less severe fading scenario ( $\kappa=4.5, \mu=4.5)$, as illustrated in Figure $2 \mathrm{~b}$, more terms are required for a better precision (in this case, a total number of $W^{2}=676$ terms is used).

An important characteristic of the proposed approximation is that it converges to the exact PEP for high SNR values, even using a small number of terms in the series.

\subsection{Lower bounds}

A classical approach to derive approximations for analytical functions is the use of bounds. Thus, the authors have derived lower and upper bounds for the PEP expression to be used in the evaluation of the modulation diversity in $\kappa-\mu$ fading. In this article, two different lower bounds were derived. The first lower bound (referred to as Lower Bound $A$ ) is obtained truncating the PEP series form, presented in (21), to only one term (i.e., $m=0$ and $n=0$ ). In this case, (21) can be rewritten as

$$
\begin{aligned}
P(s \rightarrow \hat{s}) \geq & \frac{\exp [-2 \mu \kappa] \mathrm{B}\left(2 \mu+\frac{1}{2}, \frac{1}{2}\right)}{2 \pi\left[\left(1+c_{I}\right)\left(1+c_{Q}\right)\right]^{\mu}} \\
& \times F_{1}\left(\frac{1}{2}, \mu, \mu, 2 \mu+1 ; \frac{1}{1+c_{I}}, \frac{1}{1+c_{Q}}\right) .
\end{aligned}
$$

Another lower bound can be derived replacing the exponential functions of (16) by their equivalent power series ([22], (1.211)) and applying ([22], (3.211)). After the analytical manipulations, the PEP function can be rewritten as

$$
\begin{aligned}
P(s \rightarrow \hat{s})= & \frac{\mathrm{B}\left(2 \mu+\frac{1}{2}, \frac{1}{2}\right)}{2 \pi\left[\left(1+c_{I}\right)\left(1+c_{Q}\right)\right]^{\mu}} \\
& \times \sum_{m=0}^{\infty} \sum_{n=0}^{\infty} \frac{(-1)^{m+n}}{m ! n !}\left(\frac{\mu \kappa c_{I}}{1+c_{I}}\right)^{m}\left(\frac{\mu \kappa c_{Q}}{1+c_{Q}}\right)^{n} \\
& \times F_{1}\left(\frac{1}{2}, \mu+m, \mu+n, 2 \mu+1 ; \frac{1}{1+c_{I}}, \frac{1}{1+c_{Q}}\right) .
\end{aligned}
$$

Using the fact that the Appell hypergeometric function in (23) converges to 1 at high SNR values ${ }^{\mathrm{a}}$ and transforming the series again to exponential functions, the PEP lower bound (referred to as Lower Bound B) can be written as

$$
\begin{aligned}
P(s \rightarrow \hat{s}) \geq & \frac{\mathrm{B}\left(2 \mu+\frac{1}{2}, \frac{1}{2}\right)}{2 \pi\left[\left(1+c_{I}\right)\left(1+c_{Q}\right)\right]^{\mu}} \\
& \times \exp \left[-\mu \kappa\left(\frac{c_{I}}{1+c_{I}}+\frac{c_{Q}}{1+c_{Q}}\right)\right] .
\end{aligned}
$$



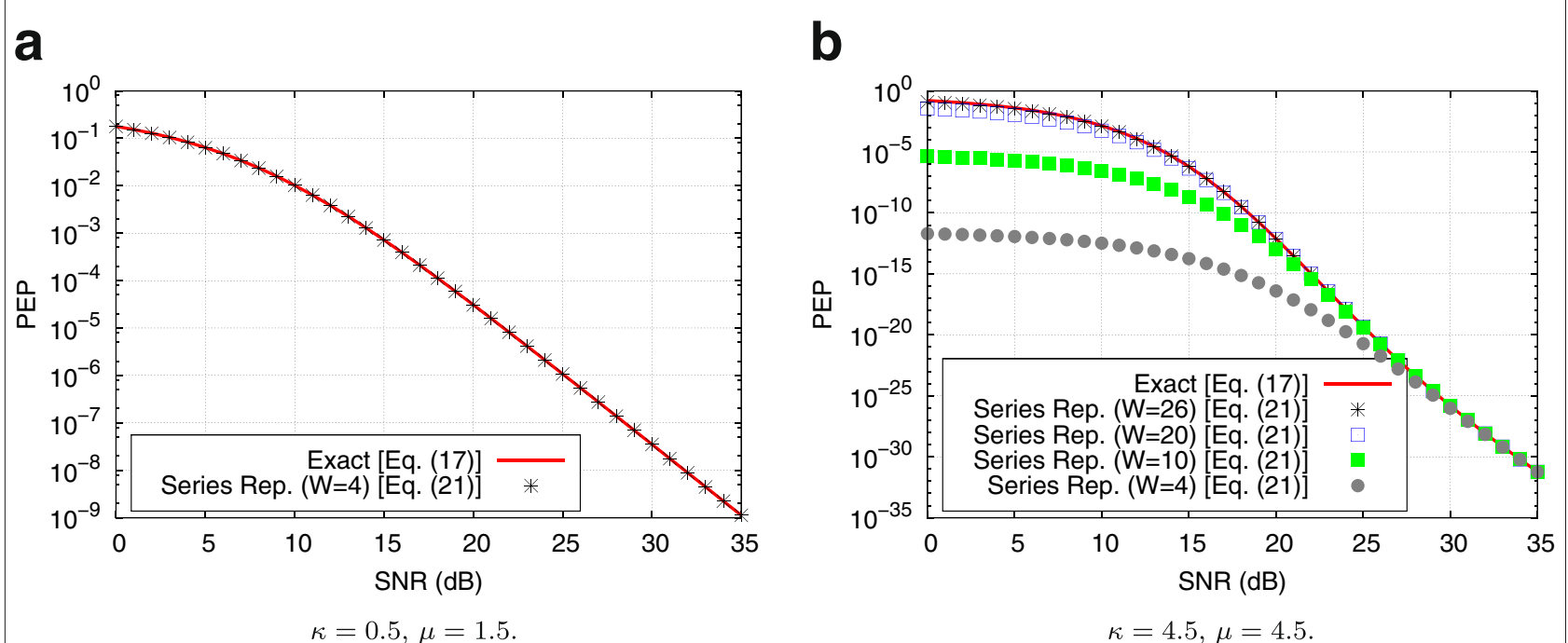

Figure 2 Series approximation for the PEP considering the randomly chosen symbols $0.3162+\mathbf{0 . 9 4 8 7} \boldsymbol{j}$ and $\mathbf{0 . 9 4 8 7}-\mathbf{0 . 3 1 6 2 j}$. The figure presents the performance of the series approximation proposed in (20) for the PEP of modulation diversity.

The lower bound presented in (24) is much simpler than the one presented in (22), since it does not require the computation of the Appell hypergeometric function.

\subsection{Upper bounds}

Two PEP upper bounds are proposed to evaluate the modulation diversity system. The first upper bound is obtained assuming that $\left(1+c_{I}\right)$ and $\left(1+c_{Q}\right)$ in the denominator of (23) converge, respectively, to $c_{I}$ and $c_{Q}$ at high SNR values, the $F_{1}(\cdot)$ function converges to 1 at high SNR values, and the series become exponential functions. Since, for low SNR values, $\left[\left(1+c_{I}\right)(1+\right.$ $\left.\left.c_{Q}\right)\right]^{\mu} \gg\left(c_{I} c_{Q}\right)^{\mu}$ and $\frac{\left[\left(1+c_{I}\right)\left(1+c_{Q}\right)\right]^{\mu}}{\left(c_{I} c_{Q}\right)^{\mu}} \gg F_{1}(\cdot)$ function, then (23) becomes an upper bound and can be rewritten as

$$
\begin{aligned}
P(s \rightarrow \hat{s}) \leq & \frac{\mathrm{B}\left(2 \mu+\frac{1}{2}, \frac{1}{2}\right)}{2 \pi c_{I}^{\mu} c_{Q}^{\mu}} \\
& \times \exp \left[-\mu \kappa\left(\frac{c_{I}}{1+c_{I}}+\frac{c_{Q}}{1+c_{Q}}\right)\right] .
\end{aligned}
$$

Furthermore, if $\kappa \rightarrow 0$ and $\mu=m$, then (25) coincides with the PEP upper bound for Nakagami- $m$ fading channels presented in ([11], Eq.(13)).

The second upper bound is based on the use of the Chernoff bound (i.e., $Q(x) \leq \frac{1}{2} e^{-\frac{x^{2}}{2}}$ ). It is obtained substituting the Chernoff bound into (12) and performing the integration in $\alpha_{I}$ and $\alpha_{Q}$. Finally, the PEP can be upper bounded by (26)

$$
\begin{aligned}
P(s \rightarrow \hat{s}) \leq & \frac{\mu^{2 \mu}(1+\kappa)^{2 \mu}}{2} \\
& \times\left[\left(\frac{\gamma d_{I}^{2}}{4}+\mu(1+\kappa)\right)\left(\frac{\gamma d_{Q}^{2}}{4}+\mu(1+\kappa)\right)\right]^{-\mu} \\
& \times \exp \left[-\gamma \kappa \mu\left(\frac{d_{I}^{2}}{d_{I}^{2} \gamma+4(1+\kappa) \mu}\right.\right. \\
& \left.\left.+\frac{d_{Q}^{2}}{d_{Q}^{2} \gamma+4(1+\kappa) \mu}\right)\right]
\end{aligned}
$$

\subsection{Performance evaluation of the PEP bounds}

The proposed bounds serve as approximations for the PEP function and are used to optimize the $\theta$ angle. However, each proposed approximation exhibits a different performance when compared to the exact PEP value, as well as different complexity.

A performance comparison of the proposed lower and upper bounds for the PEP is presented in Figure 3. In the evaluation different values of $\kappa$ and $\mu$ and the constellation symbols $0.3162+0.9487 j$ and $0.9487-0.3162 j$ are considered.

As can be seen in Figure 3, the Lower Bound B is more accurate than the other bounds. Furthermore, it is relatively simpler than the other bounds, making its adoption quite attractive. However, an important aspect to note is 

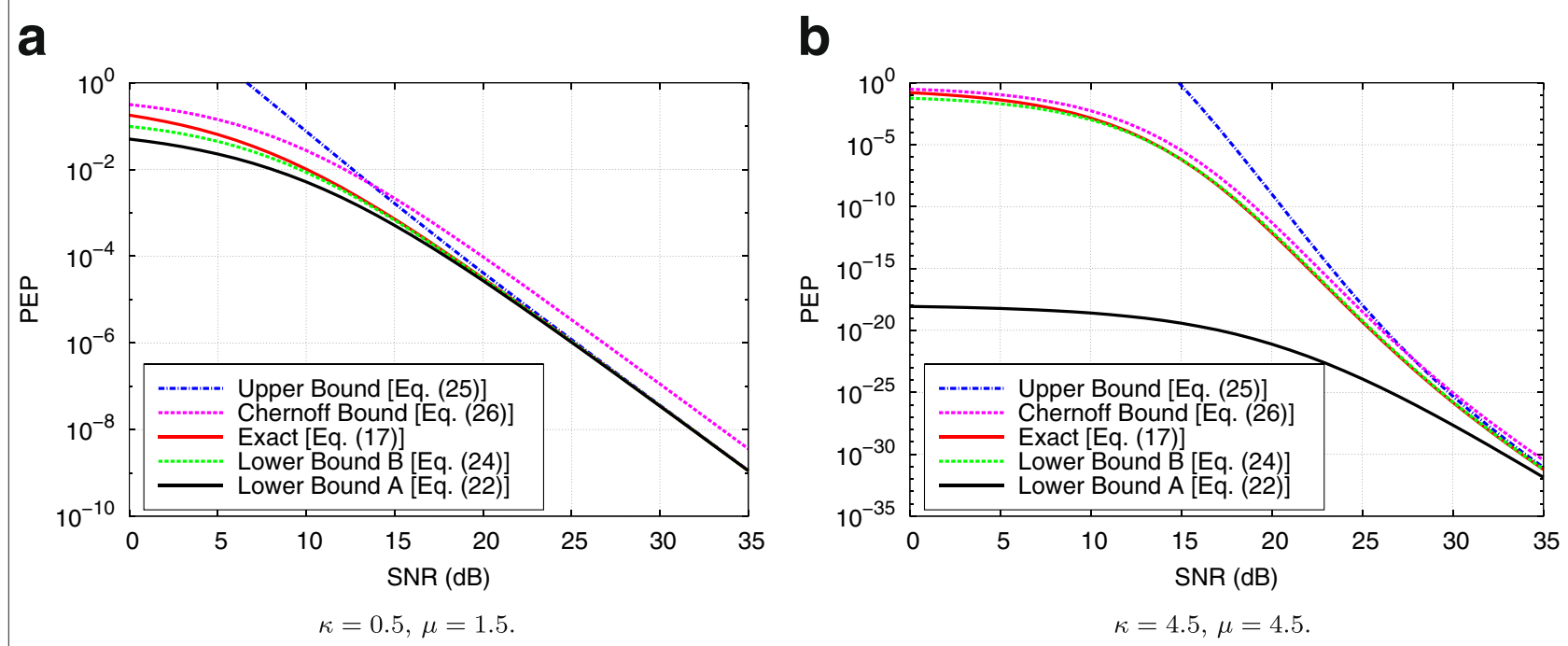

Figure 3 PEP bounds considering the symbols $\mathbf{0 . 3 1 6 2}+\mathbf{0 . 9 4 8 7} \mathbf{j}$ and $\mathbf{0 . 9 4 8 7} \mathbf{- 0 . 3 1 6 2 j}$. Figure 3 depicts a comparison among the proposed PEP approximations (namely Upper Bound, Chernoff Bound, Exact, Lower Bound A and Lower Bound B) for $\kappa-\mu$ channels. The same symbols and fading scenarios from Figure 2 are used in Figure 3.

that, whenever a lower bound is used as a PEP approximation, the union bound becomes an approximation and not an upper bound anymore.

\section{Results}

The adoption of the $\kappa-\mu$ fading model allows the evaluation of communication systems in channel conditions which are not covered by other channel models, such as the Nakagami- $m$. The flexibility provided by the $\kappa-\mu$ distribution is adequate to evaluate the performance of modulation diversity. This section presents the performance analysis of the diversity modulation technique for a $\kappa-\mu$ fading channel. Numerical evaluations and Monte Carlo simulations were performed, respectively, to optimize the $\theta$ angle in (13) and (14), and to verify the gains obtained by the modulation diversity scheme considering different channel parameters and PEP expressions.

\subsection{Evaluation of the optimum rotation angle $\theta$}

The performance of the modulation diversity technique is directly affected by the constellation rotation angle $\theta$, requiring its optimization, to obtain the value of $\theta$ that generates the lowest overall SER. The evaluation of the optimal rotation angle is accomplished by replacing the different PEP expressions in (9). The evaluation was performed considering QPSK and 16-QAM constellations and two different channel scenarios: (a) severe fading conditions $(\kappa=0.1, \mu=0.25)$ and (b) typical fading conditions ( $\kappa=1.5, \mu=1.75)$ [25].

Figures 4 and 5 depict the SER of the modulation diversity system (with QPSK and 16-QAM constellations) for a $\kappa-\mu$ fading channel. As can be seen in the figures, the optimal angle depends on the constellation order and the channel characteristics. Moreover, the SER curves are symmetric with respect to the angle $45^{\circ}$, since analogous constellations are generated regardless of the rotation direction (i.e., clockwise or counterclockwise).

Comparing Figures 4 and 5, one can note that the overall performance of the PEP approximations depends on the fading parameters $\kappa$ and $\mu$. For severe channel conditions (i.e., Figures $4 \mathrm{a}$ and $5 \mathrm{a}$ ), the Chernoff bound presents the worst performance considering the SER approximation. One can note that the other bounds exhibit a similar performance to the Exact PEP calculation.

On the other hand, in typical fading scenarios (i.e., Figures $4 \mathrm{~b}$ and $5 \mathrm{~b}$ ), the upper bound shown in (25) performs worse than the Chernoff bound. The Exact, Series and Simulation curves are indistinguishable, and the Lower Bound $B$ curve is very close to them. Finally, the Lower Bound $A$ approximation has the worst performance when compared to the Exact PEP.

An overview of the optimum rotation angle in modulation diversity systems subject to $\kappa-\mu$ fading is shown in Figure 6 which contains the curves of the optimal $\theta$ as a function of the parameters $\kappa$ and $\mu$ and considering the use of QPSK and 16-QAM constellations, respectively. The optimization process was conducted using the Lower Bound B approximation, due to its simplicity and accuracy.

As can be seen in Figure 6a, the optimum angle in a QPSK constellation assumed values in the range $27^{\circ}$ to $32^{\circ}$. The largest optimum angle value $\left(31.4^{\circ}\right)$ is achieved 


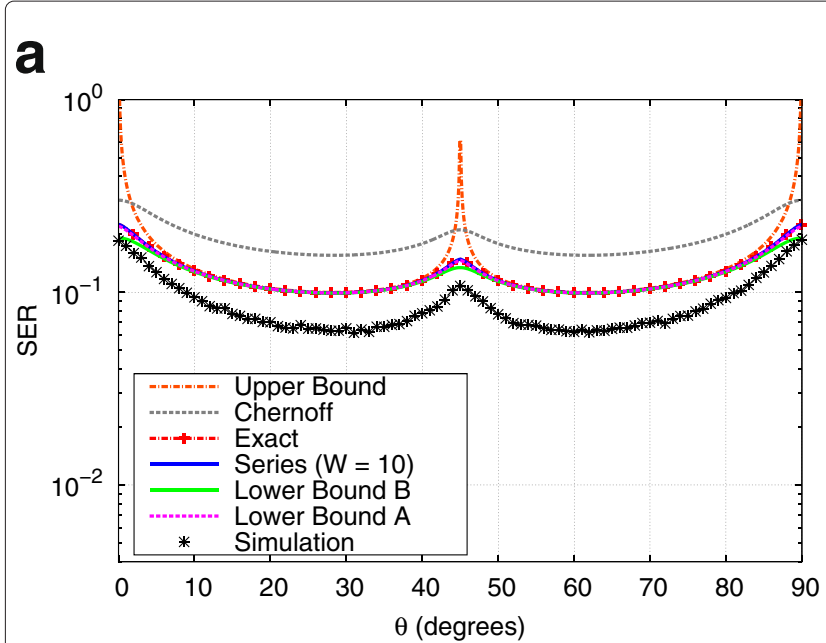

$$
\kappa=0.1, \mu=0.25, \mathrm{SNR}=20 \mathrm{~dB}\left(\text { Optimum } \theta=28.32^{\circ}\right) .
$$

b

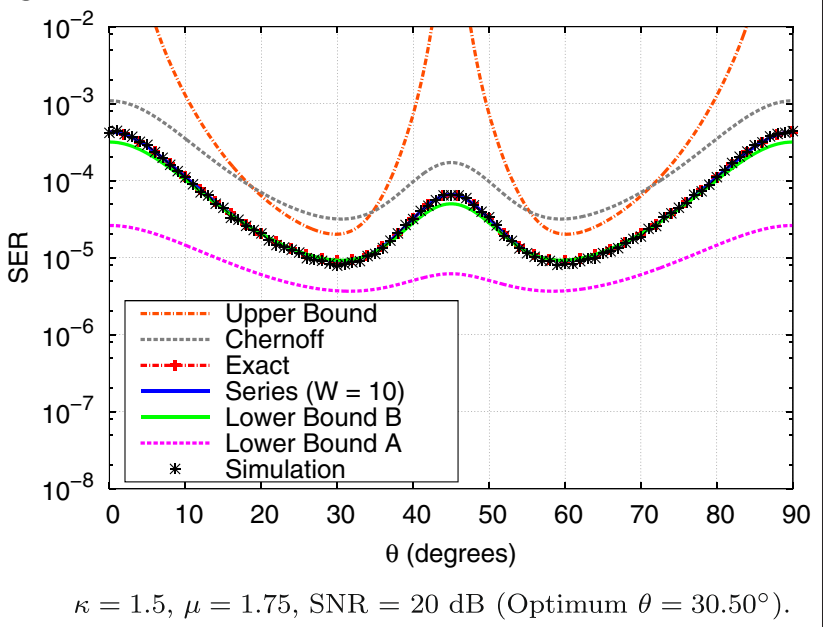

Figure 4 SER of the QPSK modulation diversity system as a function of the rotation angle $\boldsymbol{\theta}$. The simulated and approximated SER values for a modulation diversity system (with QPSK) are shown in the figure. Two fading scenarios were used in the evaluation: (a) severe fading $(\kappa=0.1$, $\mu=0.25)$ and (b) typical fading $(\kappa=1.5, \mu=1.75)$. The fading scenarios were evaluated for an $\mathrm{SNR}=20 \mathrm{~dB}$.

at high values of $\mu$ and low values of $\kappa$ (i.e., the fading conditions are less severe). In contrast, the lowest optimum angle value $\left(27.8^{\circ}\right)$ should be used for low $\kappa$ and $\mu$ values (i.e., in very severe channel fading). Furthermore, other intermediate values should be selected according to the channel fading conditions, using the results shown in Figure 6a.

Figure $6 \mathrm{~b}$ presents the optimum rotation angle $\theta$ for the 16-QAM constellations as a function of the channel fading parameters. As shown in the figure, there are abrupt transitions in the graphic, the result of small changes in the minimum values of the system SER (i.e., minor changes in the sidelobes of the SER curves-refer to Figure 5).

If a Nakagami- $m$ channel fading model is considered (i.e., $\kappa \rightarrow 0$ and $\mu=m$ ), the optimum $\theta$ values are confirmed by the values presented in ([11], Table one) (for QPSK constellations), which confirms the precision of the Lower Bound B approximation.

\subsection{Evaluation of the execution time}

In addition to the SER evaluation, the authors evaluated the average execution time of 900 union bound calculations, considering the different proposed PEP

\section{a}

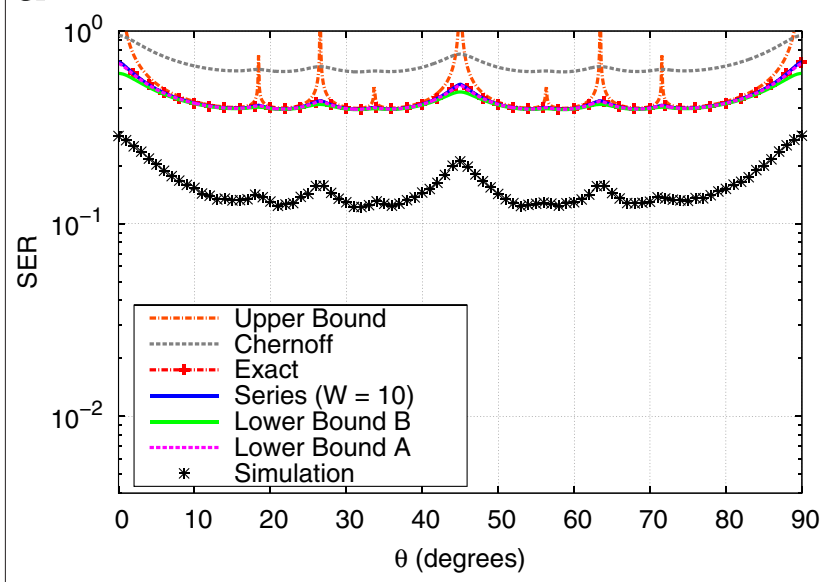

$\kappa=0.1, \mu=0.25, \mathrm{SNR}=26 \mathrm{~dB}\left(\right.$ Optimum $\left.\theta=31.51^{\circ}\right)$. b

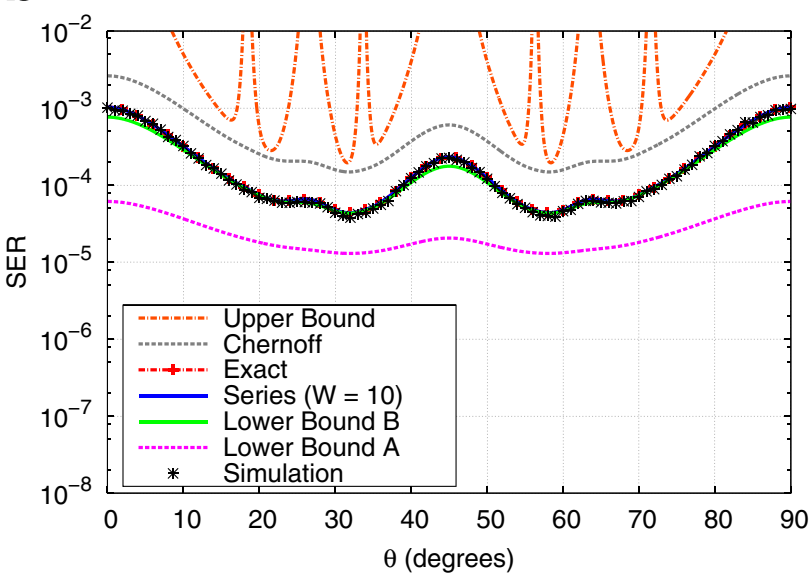

$\kappa=1.5, \mu=1.75, \mathrm{SNR}=26 \mathrm{~dB}\left(\right.$ Optimum $\left.\theta=31.81^{\circ}\right)$.

Figure 5 SER of the 16-QAM modulation diversity system as a function of the rotation angle $\boldsymbol{\theta}$. The simulated and approximated SER values for a modulation diversity system (with 16-QAM) are presented in the figure. The same fading scenarios from Figure 4 were used. The fading scenarios were evaluated for an $\mathrm{SNR}=26 \mathrm{~dB}$. 


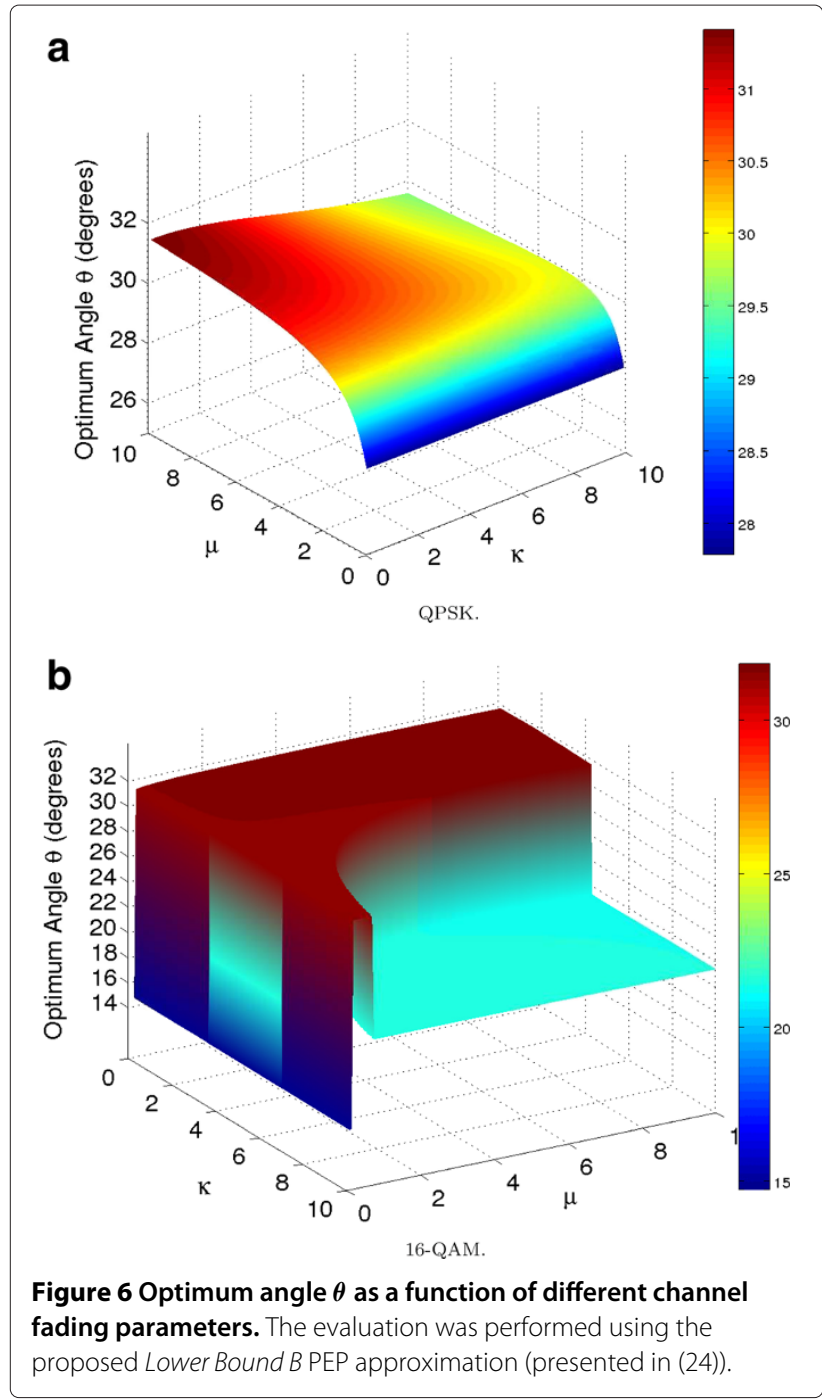

approximations. Furthermore, each round of the experiment was executed 30 times, in order to provide statistical inferences. Table 1 shows the execution times for the QPSK and 16-QAM union bounds considering the proposed PEP expressions.

As can be seen in the table, considering the most accurate approximations (as discussed in Section 4.1), the Lower Bound B presented the lowest execution time. Its running time is slightly above the Chernoff and Upper Bound approximations, but presents an improved accuracy, and is attractive to use in the rotation optimization process.

\subsection{Evaluation of the System Symbol Error Rate}

Based on the use of the of the Lower Bound B approximation, this section presents the SER evaluation of communication systems that use the modulation diversity technique for $\kappa-\mu$ fading channels. Monte Carlo
Table 1 Average execution time for 900 calculations of the union bound considering the proposed PEP approximations

\begin{tabular}{|c|c|c|c|}
\hline Constellation & Fading & PEP expression & Exec. time (sec.) \\
\hline \multirow[t]{12}{*}{ QPSK } & Severe & Upper bound & 0.020 \\
\hline & & Chernoff & 0.022 \\
\hline & & Exact & 0.922 \\
\hline & & Series & 148.490 \\
\hline & & Lower bound A & 1.064 \\
\hline & & Lower bound B & 0.023 \\
\hline & Typical & Upper bound & 0.026 \\
\hline & & Chernoff & 0.019 \\
\hline & & Exact & 1.701 \\
\hline & & Series & 168.540 \\
\hline & & Lower bound A & 1.985 \\
\hline & & Lower bound B & 0.031 \\
\hline \multirow[t]{12}{*}{ 16-QAM } & Severe & Upper bound & 0.170 \\
\hline & & Chernoff & 0.124 \\
\hline & & Exact & 17.924 \\
\hline & & Series & 2956.030 \\
\hline & & Lower bound A & 20.827 \\
\hline & & Lower bound B & 0.167 \\
\hline & Typical & Upper bound & 0.172 \\
\hline & & Chernoff & 0.126 \\
\hline & & Exact & 30.335 \\
\hline & & Series & 3226.570 \\
\hline & & Lower bound A & 34.965 \\
\hline & & Lower bound B & 0.187 \\
\hline
\end{tabular}

Table 1 presents the average execution time for 900 calculations of the union bound considering the proposed PEP approximations and simulations. Two fading scenarios were used in the evaluation: (a) severe fading $(\kappa=0.1$, $\mu=0.25)$ and (b) typical fading ( $\kappa=1.5, \mu=1.75)$.

simulations were performed to evaluate the efficiency as modulation diversity is used for fading. The system dynamically adapts the rotation angle according to the channel SNR using the golden section search method ([26], Section 10.2). The same channel and system parameters used in the experiments of Section 4.1 were adopted (Figures 4 and 5).

Symbol error rates for the QPSK and 16-QAM systems, with and without the use of modulation diversity, are depicted, respectively, in Figures 7 and 8. In addition to the simulated values, the union bound and nearest neighbor curves are also plotted to be compared to the simulated values. The union bound curve (Equation (9)) is calculated using the exact PEP expression (Equation (17)), while the nearest neighbor curve (Equation (10)) is calculated with the Lower Bound B approximation (Equation (24)). 

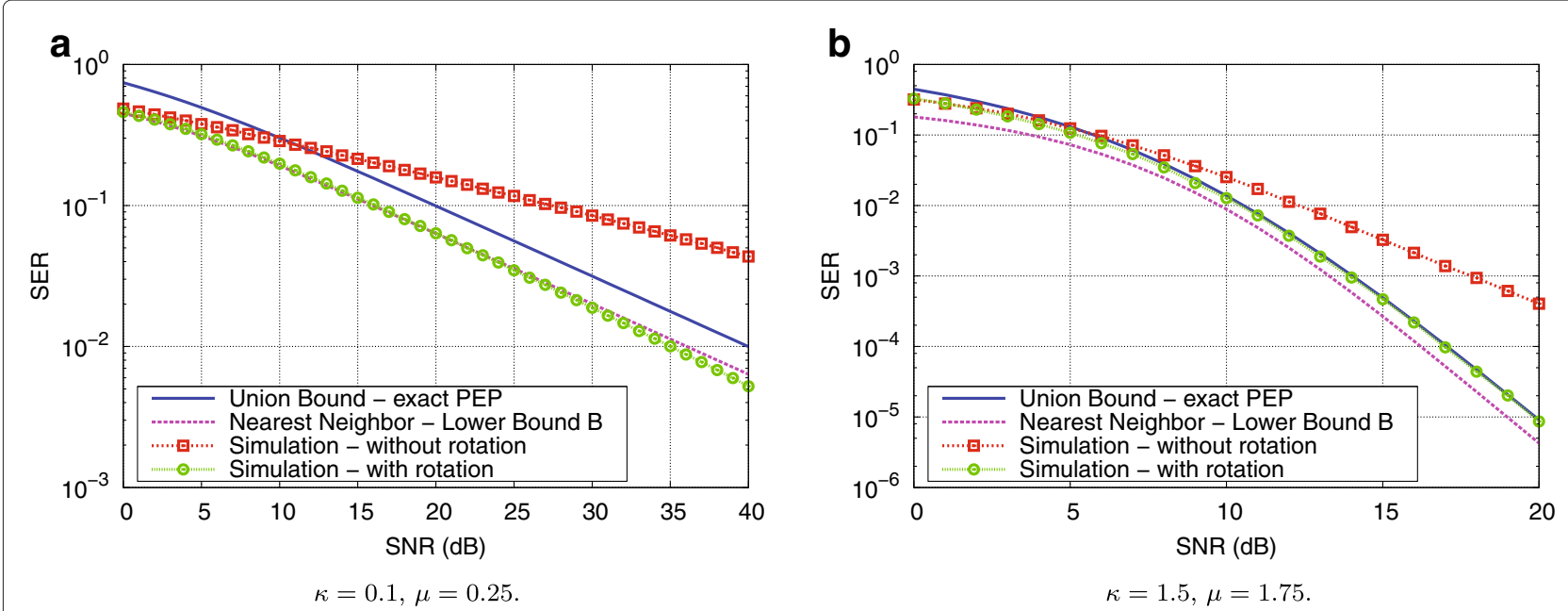

Figure 7 SER of a QPSK system with and without rotation in severe and typical fading conditions.

In a conventional transmission, the fading peaks can completely degrade the information of the transmitted symbols (in-phase and quadrature components). However, using the modulation diversity technique, the symbol components are transmitted at different instants of time, creating a redundancy between those components. In this context, the gain provided by the modulation diversity is higher under severe fading conditions, but it does not affect the system performance when the signals are transmitted in AWGN channels, since the Euclidean distance between the symbols remains constant regardless of the rotation angle $\theta$. This aspect can be verified in Figures 7 and 8 , the rotated constellation outperforms the reference system (i.e., without rotation). However, one can note that the gain provided by this technique decreases as the fading severity is reduced (i.e., as the values of $\kappa$ and $\mu$ are increased).
For the QPSK system, the modulation diversity gain is $16.86 \mathrm{~dB}$ (for a SER of $4.35 \times 10^{-2}$ ) considering severe fading conditions $(\kappa=0.1, \mu=0.25)$, and is $4.80 \mathrm{~dB}$ (for a SER of $4.04 \times 10^{-4}$ ) in a typical fading scenario $(\kappa=1.5, \mu=1.75)$. On the other hand, for the 16-QAM system, a gain of $11.28 \mathrm{~dB}$ (for a SER of $9.30 \times 10^{-2}$ ) is obtained in severe fading, while in typical fading, the modulation diversity system has a gain of $3.74 \mathrm{~dB}$ (for a SER of $1.34 \times 10^{-3}$ ).

Another important aspect to note is that the union bound is not a good approximation for channels subject to severe fading conditions, but it becomes a suitable approximation for better channel conditions. Instead, the nearest neighbor, with the Lower Bound $B$ PEP, fits well in severe fading (e.g., Figure 7a), but becomes a lower bound in typical fading scenario (e.g., Figure $7 \mathrm{~b})$.
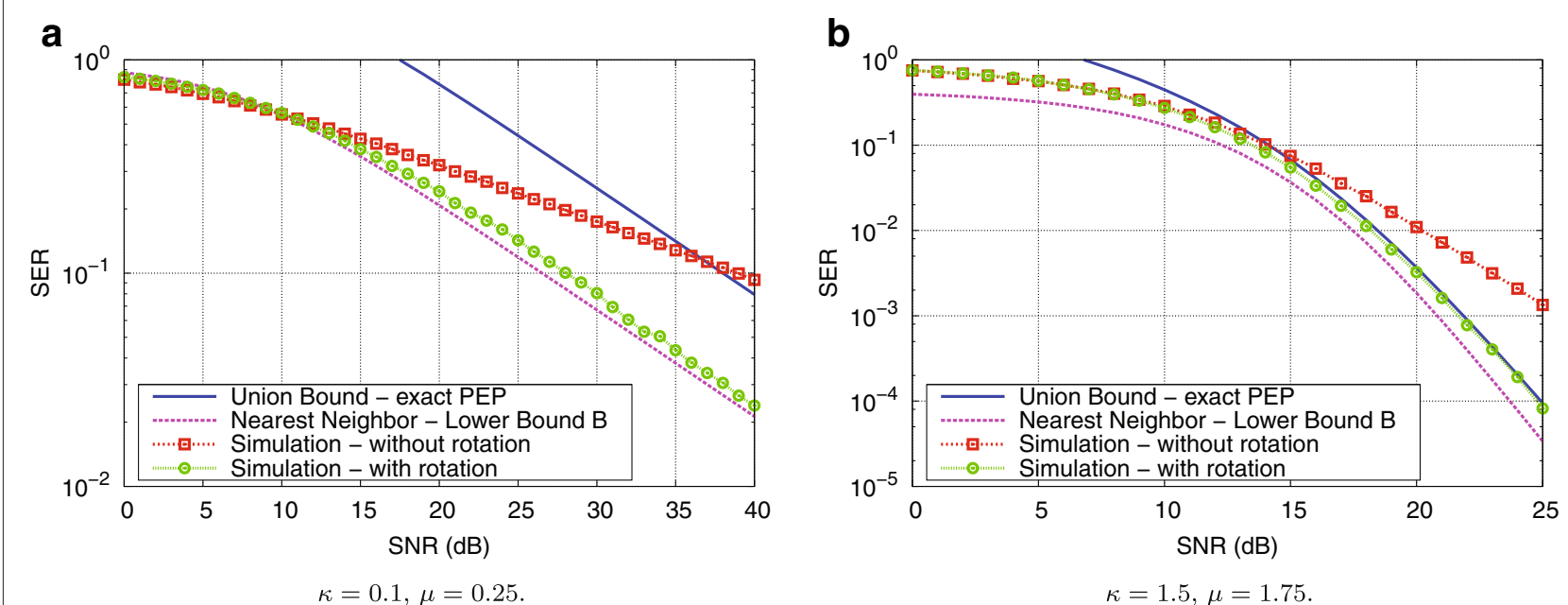

Figure 8 SER of a 16-QAM system with and without rotation in severe and typical fading conditions. 


\section{Performance evaluation of the modulation diversity technique in time correlated channels}

The previous evaluation of the modulation diversity technique considered that the in-phase (I) and quadrature (Q) components are independently affected by the fading. That assumption is based on the fact that the interleaving depth (i.e., the temporal shift between a pair of interleaved symbols, denoted by $k$ ) is larger than the channel coherence bandwidth. However, in an actual scenario, the channel conditions constantly change, and the perfect channel state information may not be available, preventing the system from dynamically adapt the interleaving depth. Moreover, the coherence bandwidth changes according to the Doppler frequency, which depends on the relative velocity between the transmitter and the receiver. Therefore, some degree of temporal correlation between the fading coefficients appears, affecting the performance of the modulation diversity technique.

This section presents an evaluation of the modulation diversity assuming a time correlated channel. An analysis of the influence of the correlation on the rotation angle and on the performance of the technique is also shown.

\subsection{Generation of a time correlated $\kappa-\mu$ fading channel}

The first challenge to be faced in the proposed evaluation is the generation of a time correlated $\kappa-\mu$ fading. The developed $\kappa-\mu$ time correlated fading generator is based on the adaptation of the classical time correlated Rayleigh fading generator, on the properties of the Gaussian processes and on the $\kappa-\mu$ fading physical model.

According that model [17], the received signal is a composition of multiple clusters, each one consisting of scattered waves of identical powers and of a dominant component of arbitrary power. Thus, the envelope $R$ of the $\kappa-\mu$ distribution can be defined in terms of its in-phase and quadrature components, as follows

$$
R^{2}=\sum_{i=1}^{n}\left(X_{i}+p_{i}\right)^{2}+\sum_{i=1}^{n}\left(Y_{i}+q_{i}\right)^{2}
$$

in which $X_{i}$ and $Y_{i}$ are independent Gaussian random processes with means $E\left[X_{i}\right]=E\left[Y_{i}\right]=0$ and variances $V\left[X_{i}\right]=V\left[Y_{i}\right]=\sigma^{2}, p_{i}$ and $q_{i}$ are, respectively, the mean values of the in-phase and quadrature components of the $i$ th cluster and $n$ is the number of clusters.

As discussed in Section 2, the parameters $\kappa$ and $\mu$ define the shape of the distribution, with $\kappa$ representing the ratio between the total power of the dominant components and the total power of the scattered components, analytically defined as follows [17]

$$
\kappa=\frac{\sum_{i=1}^{n}\left(p_{i}^{2}+q_{i}^{2}\right)}{2 \mu \sigma},
$$

and $\mu$ is a real extension of $n$, given by [17]

$$
\mu=\frac{E^{2}\left[R^{2}\right]}{V\left[R^{2}\right]} \times \frac{1+2 \kappa}{(1+\kappa)^{2}} .
$$

The parameter $\mu$ extends the original meaning of the parameter $n$ to include some specific channel characteristics, such as [17]: (a) non-zero correlation among the clusters of multipath components; (b) non-zero correlation between the in-phase and quadrature components within each cluster; and (c) the non-Gaussian nature of the in-phase and quadrature components of each cluster of the fading signal, among other characteristics.

Based on the physical model of the $\kappa-\mu$ channel, Figure 9 presents the block diagram of the proposed fading generator (with $p_{i}, q_{i}=m, \forall 1 \leq i \leq n$ ). As can be seen in the figure, the resultant correlated $\kappa-\mu$ process is composed by a sum of $n$ correlated Rician processes (with each process representing a cluster of multipath waves, containing a dominant component).

The first step performed by the proposed system is the generation of a time correlated Rayleigh fading. Different techniques are available in the literature to generate time correlated Rayleigh fading channels, including the well-known techniques of Smith spectrum [27] and sum of sinusoids [28]. The corresponding normalized autocorrelation function for the Rayleigh fading is given by [29]

$$
R(\tau)=J_{0}\left(2 \pi f_{D} \tau\right)
$$

in which $\tau$ is the time shift delay and $f_{D}$ is the maximum Doppler frequency. The power spectrum density of a time-correlated Rayleigh fading is given by the classical Jake's Doppler spectrum [27,30], as follows

$$
S(f)=\left\{\begin{array}{rr}
\frac{1}{\pi f_{D} \sqrt{1-\left(\frac{f}{f_{D}}\right)^{2}},} & |f| \leq f_{D}, \\
0, & |f|>f_{D},
\end{array}\right.
$$

in which $f$ is the frequency shift relative to the carrier frequency.

The Rayleigh random variable is transformed, by a suitable function, to produce the Rice fading. Since the Rayleigh fading is a circularly symmetric Gaussian process, its statistics can be modified, without losing its Gaussian characteristics. Thus, the Rice fading is obtained from the complex Gaussian process $\alpha=X+j Y$, in which the real and imaginary components have mean $m$ and standard deviation $s$, i.e., $X, Y \sim N\left(m, s^{2}\right)$, given by [31]

$$
\begin{gathered}
m=\sqrt{\frac{K}{2(K+1)}} \\
s=\sqrt{\frac{1}{(K+1)}},
\end{gathered}
$$

in which $K$ (the Rice factor) is the ratio of the lineof-sight (LOS) the non-line-of-sight (NLOS) component, 


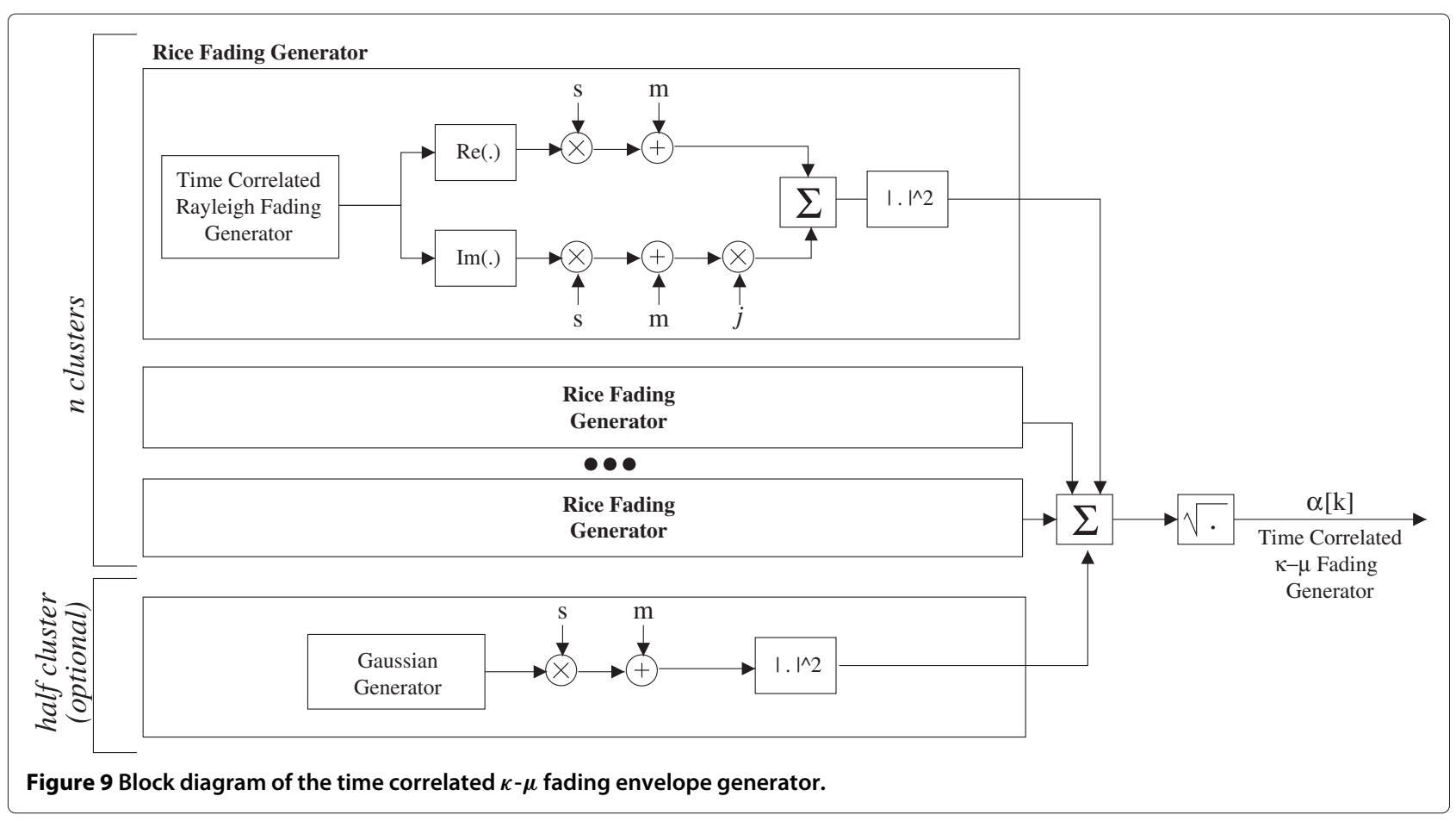

$K=0$ corresponds to a Rayleigh fading channel and $K \rightarrow \infty$ corresponds to a non-fading (i.e., constant) channel.

The Rice fading generation process must be repeated $n$ times, with $n$ being the corresponding integer value of the channel parameter $\mu$. An optional Gaussian generator can be used for half-integer values of the parameter $\mu$.

This limitation requires the adaptation of the previously used channel scenarios to new values, as follows: (a) the severe fading conditions $(\kappa=0.1, \mu=0.5)$ and (b) typical fading conditions $(\kappa=1.5, \mu=2.0)$.

Finally, the squared norm of the Rician processes are added and a square root is applied to generate the time correlated $\kappa-\mu$ process. A normalization of the fading samples is also required.

Figure 10 presents the histograms of the envelopes for the two $\kappa-\mu$ fading scenarios (generated using the developed system) and their corresponding theoretical pdfs. As can be seen, the histograms fit well with the theoretical pdfs.

The normalized autocorrelation function, for both scenarios, was calculated using the generated fading samples. Figure 11 shows the autocorrelation for the fading scenarios, considering a sampling frequency of 270833 symbols/second (analogous to the one used in GSM-Global System for Mobile Communications), and two different Doppler frequencies.

As expected, the presence of dominant components and a non-unitary number of clusters (i.e., determined by $\mu$ ), create a correlation among the fading samples. As a result, the channel does not become uncorrelated (instead, the correlation reduces or increases according to the temporal separation of the samples). Finally, the generated fading samples are ready to be used for the evaluation of the modulation diversity technique.

\subsection{Performance over time correlated channel}

The overall performance of the modulation diversity technique for uncorrelated channels is only affected by the rotation angle of the signal constellation (that must be defined according to the channel characteristics). Correlated channels require that the interleaving depth $k$ should be carefully defined, to reduce the correlation between the interleaved channel fading samples. Therefore, the smaller the maximum Doppler frequency $f_{D}$, the larger should be the interleaving depth, requiring the analysis of the effect of the change in the interleaving depth.

Figure 12 presents the Bit Error Rate (BER) curves for a 16-QAM system as a function of the interleaving depth $k$. The evaluation was performed considering the typical fading scenario $(\kappa=1.5, \mu=2.0)$, a sampling frequency of 270833 symbols/second and three different values of SNR (10, 15, and $20 \mathrm{~dB})$. For each SNR value, three curves were generated: (a) a system without rotation $\left(0.0^{\circ}\right)$ in a correlated channel, (b) a system with rotation $\left(30.2^{\circ}\right.$ and $45.0^{\circ}$ ) in an uncorrelated channel, and (c) a system with rotation $\left(30.2^{\circ}\right.$ and $\left.45.0^{\circ}\right)$ in a correlated channel. Maximum Doppler frequencies of $100 \mathrm{~Hz}$ and $200 \mathrm{~Hz}$ were 

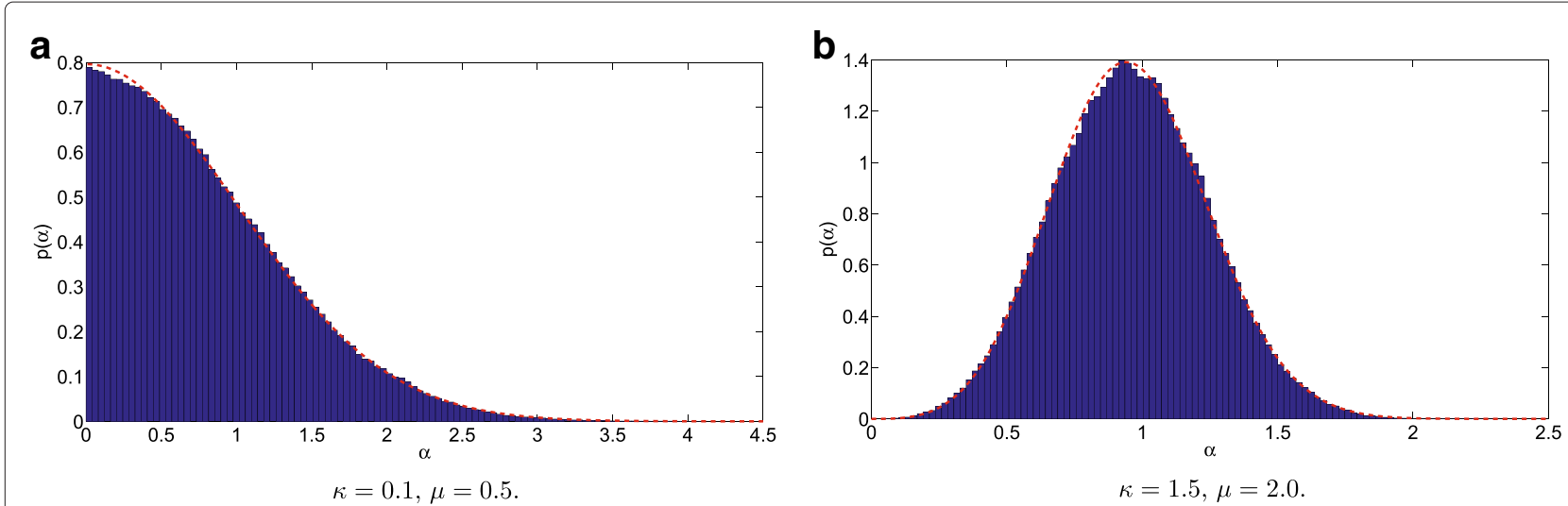

Figure 10 Histograms of two generated $\kappa-\mu$ fading channels and their corresponding theoretical pdfs (represented by the red dashed lines).

considered in the evaluation. The rotation angle for each scenario was optimized using the union bound with the proposed PEP approximation Lower Bound B (presented in (24)).

As can be seen in the figures, in correlated channels, the BER of the system with modulation diversity changes with the interleaving depth. The BER reduces as the correlation between the interleaved symbols also reduces. For instance, the minimum BER in Figure 12a is achieved when the $k$ value is approximately 1650 symbols (which is equivalent to the smallest correlation value in Figure $11 \mathrm{~b}$, $f_{D}=100 \mathrm{~Hz}$ ). Similarly, in Figure 12b, the minimum BER is obtained when $k$ is approximately 825 symbols (which is equivalent to the smallest correlation value in Figure $11 \mathrm{~b}$, $f_{D}=200 \mathrm{~Hz}$, and the double of the $100 \mathrm{~Hz}$ scenario). An important characteristic to be noted in the presented curves is that, for the minimum correlation points, the
BER of time correlated channels is smaller than the value obtained for uncorrelated channels.

In the absence of rotation $\left(0.0^{\circ}\right)$, the system becomes invariant to changes in the interleaving depth, since there is no redundancy between the I and Q interleaved components of the transmitted symbols. Finally, a channel without time correlation is equivalent to a channel with $f_{D} \rightarrow \infty$ (i.e., any pair of interleaved symbols is uncorrelated). Since the correlation among the transmitted symbols is null, the interleaving depth $k$ also does not affect the performance of the modulation diversity system.

The system BER was evaluated as a function of the rotation angle, to access the influence of the time correlation in the optimum rotation angle. Figure 13 illustrates the obtained curves for a 16-QAM system subject to a typical fading scenario $(\kappa=1.5, \mu=2.0)$, a sampling frequency
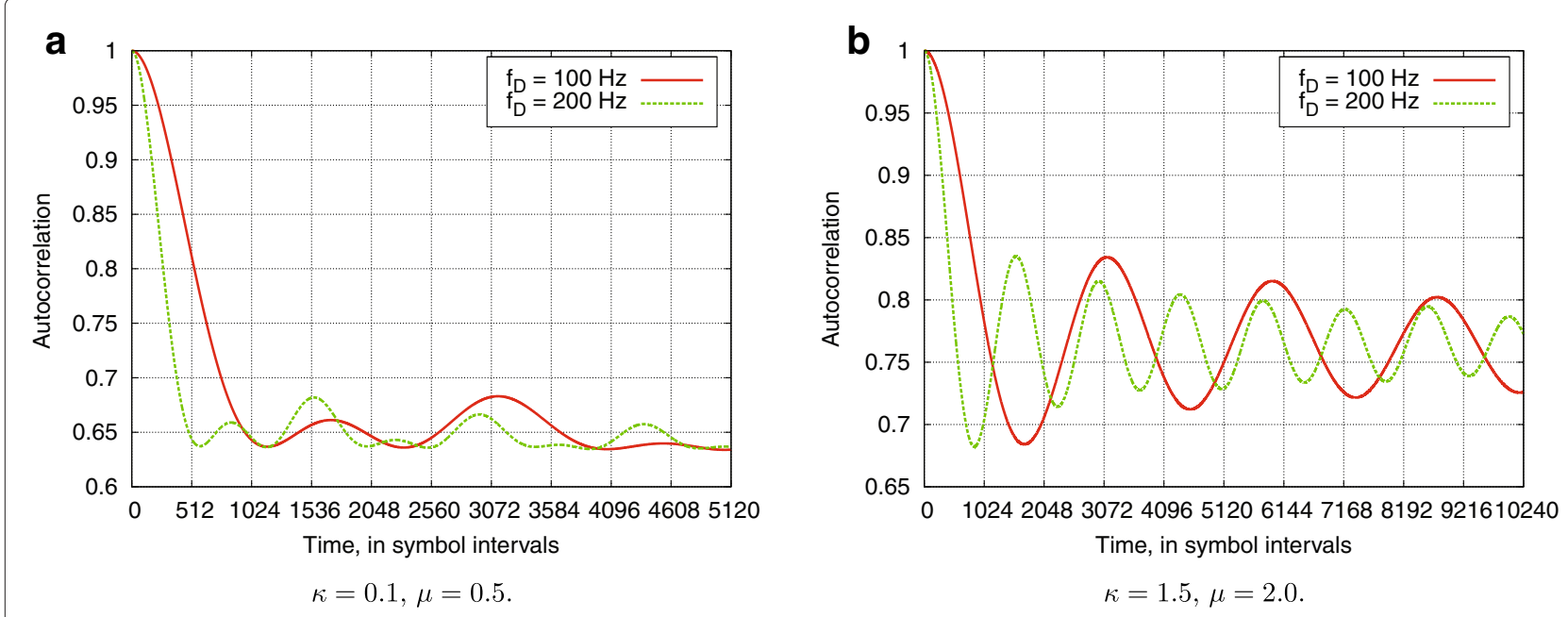

Figure 11 Normalized autocorrelation function calculated from the generated samples for both fading scenarios. 

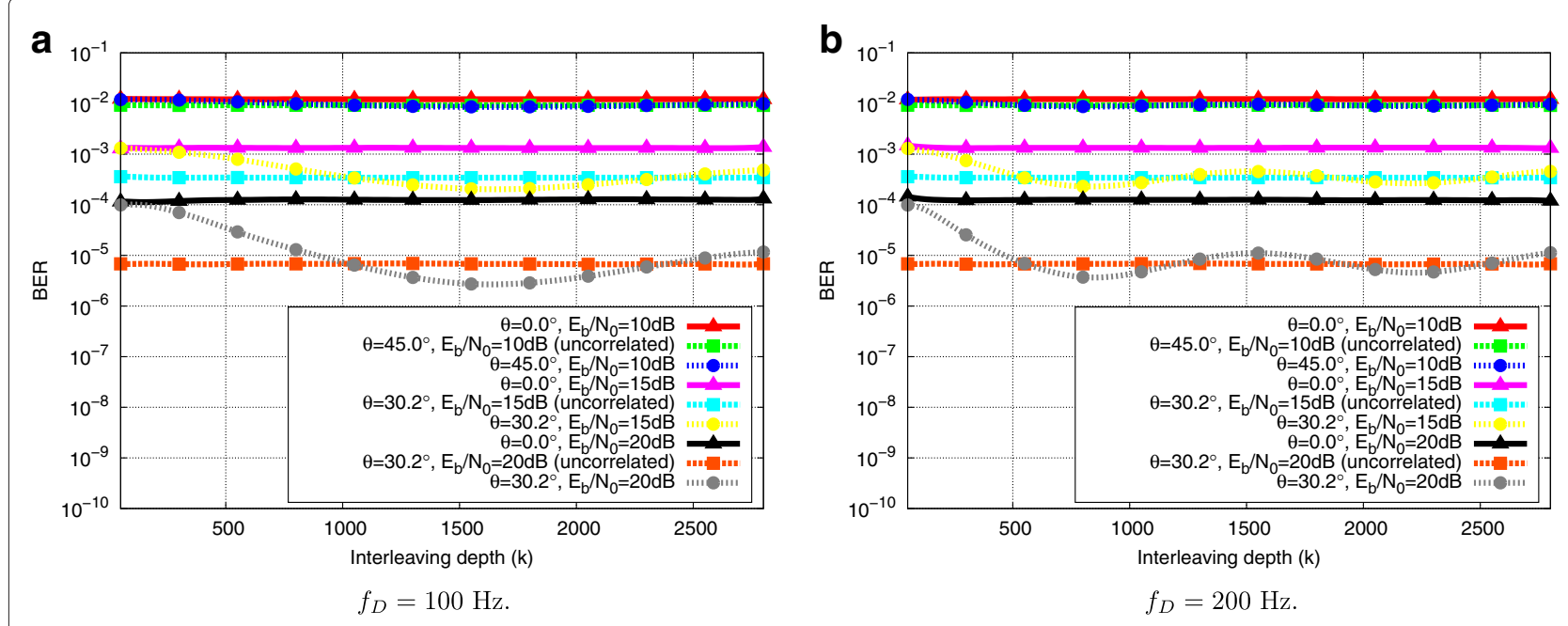

Figure 12 BER of the modulation diversity system as a function of the interleaving depth considering a 16-QAM system in a typical fading scenario $(\kappa=1.5, \mu=2.0)$, a sampling frequency of 270833 symbols/second and three different values of SNR $(10,15$, and $20 \mathrm{~dB})$.

of 270833 symbols/second, an SNR of $20 \mathrm{~dB}$, different maximum Doppler frequencies and interleaving depths.

The performance of the system for $f_{D}=100 \mathrm{~Hz}$ and $k=$ 1650 is equivalent to the performance for $f_{D}=200 \mathrm{~Hz}$ and $k=825$, since in both scenarios the system experiment the same correlation level. The figure shows the absence of significant variations in the value of the optimum angle between correlated and uncorrelated channels. Furthermore, as discussed earlier, for the minimum correlation points, the system BER for time correlated channels is smaller than the value obtained for uncorrelated channels.

Finally, Figure 14 shows the BER curves of the modulation diversity as a function of the SNR. The rotation angle used in that evaluation was obtained averaging the

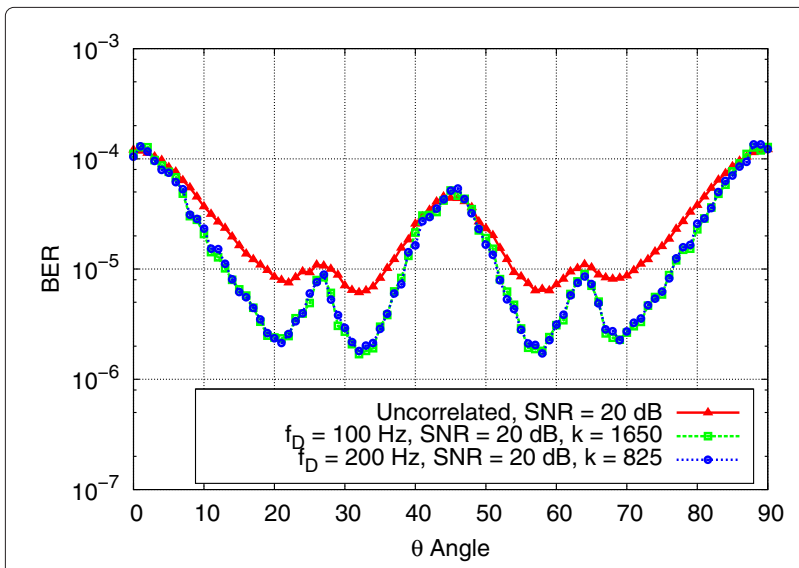

Figure 13 BER of the modulation diversity system as a function of the rotation angle. The evaluation was performed considering a 16-QAM system under a typical fading scenario ( $\kappa=1.5, \mu=2.0$ ), a sampling frequency of 270833 symbols/second, an SNR of $20 \mathrm{~dB}$ and different maximum Doppler frequencies. optimum rotation angles presented in Figure 12. In the experiments, a 16-QAM system subject to a typical fading scenario $(\kappa=1.5, \mu=2.0)$, and a sampling frequency of 270833 symbols/second (for a time correlated channel) were considered. Similar results were obtained for $f_{D}=100 \mathrm{~Hz}$ and $k=1650$, and for $f_{D}=200 \mathrm{~Hz}$ and $k=825$, therefore, only one curve is shown in the figure.

The addition of modulation diversity gives a gain improvement of $5.7 \mathrm{~dB}$ (for a BER of $10^{-5}$ ). However, the system gain increases when correlated channels are considered and an appropriate value of $k$ is defined, which represents an additional gain of approximately $1 \mathrm{~dB}$ when compared to the uncorrelated channel scenario (for the same BER value).

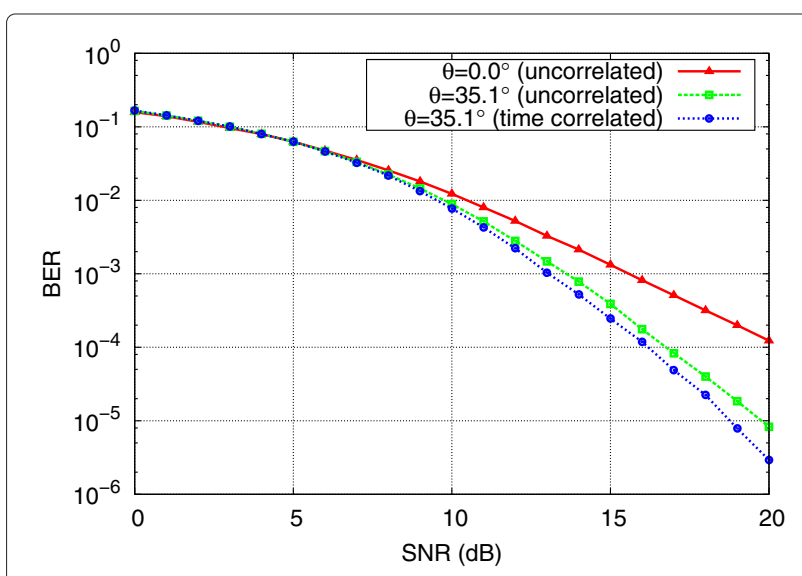

Figure $14 \mathrm{BER}$ of the modulation diversity system as a function of the SNR for a 16-QAM system under a typical fading scenario $(\kappa=1.5, \mu=2.0$ ) and for a sampling frequency of 270833 symbols/second. 
Based on the experiments, one concludes that, for time correlated channels, the interleaving depth must be carefully defined to improve the overall system performance. The correct choice of the interleaving depth reduces the system BER to lower values than the obtained in uncorrelated channels (i.e., channels with $f_{D} \rightarrow \infty$ ). Another consequence of using the optimum interleaving depth is that there are no significant changes in the optimum rotation angle.

\section{Performance evaluation of the modulation diversity technique subject to channel estimation errors}

In the previous sections, the performance of the modulation diversity system was evaluated considering the existence of ideal channel state information (CSI), i.e., the channel gain is perfectly known. In a practical implementation, the channel gain is not known and should be estimated at the receiver. The estimated values are used to compensate the fading effects on the received symbols. However, the larger the system error estimation, the larger the degradation in performance of the communication system.

Monte Carlo simulation was conducted to verify the influence of the estimation errors on the performance of the modulation diversity. Those experiments aim to investigate the impact of using classical channel amplitude and phase estimation algorithms on the optimum rotation angle value and on the overall system performance.

An analysis of the impact of the estimation errors on the modulation diversity system, as well as the results of the experiments are presented in this section. The least mean square (LMS) and the first order phase-locked loop (PLL) [32] algorithms, adopted to track the amplitude and phase of the wireless communication channel, are also described.

\subsection{Estimation algorithms}

The estimation algorithms are used to track the amplitude and phase of the channel impulse response. This allows the system to compensate the effects of the fading in the received signals, improving the overall performance. This section presents two estimators: (a) LMS, for amplitude estimation and (b) PLL, for phase estimation.

\subsubsection{Amplitude estimator}

In the evaluation, the LMS algorithm was used to estimate the amplitude of the channel impulse response. The algorithm operates by using a recursive relation, that continuously updates the estimated channel amplitude $\hat{\alpha}(n)$, as follows [32]

$$
\hat{\boldsymbol{\alpha}}(n+1)=\hat{\boldsymbol{\alpha}}(n)+\lambda \boldsymbol{s}(n) \boldsymbol{e}^{*}(n),
$$

in which $\lambda$ is the step-size parameter of the LMS algorithm, $(\cdot)^{*}$ is the complex conjugate operator and $\boldsymbol{e}(n)$ is the error signal given by

$$
\boldsymbol{e}(n)=\boldsymbol{r}(n)-\hat{\boldsymbol{\alpha}}(n) \hat{\boldsymbol{s}}(s),
$$

in which $\boldsymbol{r}(n)$ is the $n$th received signal sample, $\hat{\boldsymbol{\alpha}}_{n}$ is the $n$th estimated fading amplitude sample and $\hat{s}(s)$ is the $n$th estimated transmitted signal. During the training process $\hat{\boldsymbol{s}}(s)=\boldsymbol{s}(s)$. After the training process, the signal estimate is provided by the detector.

\subsubsection{Phase estimator}

Since the performance of the modulation diversity is affected by the constellation rotation angle, the channel phase estimation becomes a crucial aspect to be handled in the system. For the evaluation a first order PLL algorithm was used.

Similarly to the LMS algorithm, the PLL uses a recursive filter in the estimation. The phase updating is performed using the following expression

$$
\hat{\boldsymbol{\phi}}(n+1)=\hat{\boldsymbol{\phi}}(n)+\rho u_{\phi}(n),
$$

in which $\rho$ is the step of the recursive filter and $u_{\phi}(n)$ is the phase error detector, given by [33]

$$
u_{\phi}(n)=\operatorname{Im}\left[e^{-j \hat{\phi}} s^{*}(n) r(n)\right] .
$$

The PLL algorithm aims to maximize the phase likelihood function, which is obtained when the output of the phase error detector is zero. A more complete description of the PLL algorithm can be found in [33].

\subsection{Evaluation of the optimum rotation angle considering channel estimation errors}

In actual communication systems, the fading estimation algorithms are unable to perfectly track the amplitude and phase of the channel impulse response. The presence of estimation errors degrades the performance of the modulation diversity, as well as affects the value of the optimum rotation angle.

The fading estimation is independently performed on each block of symbols (using a training sequence transmitted at the beginning of each block). The larger the size of the training sequence, the better the performance of the estimator (at the cost of a reduction in the system throughput). In the performed evaluation, $20 \%$ of each block of symbols consists of training symbols (similar to the value adopted in the GSM system, that uses approximately $17.6 \%$ of the block size for training).

The performance of the LMS and PLL algorithms depend on the value of the $\lambda$ and $\rho$ parameters. Therefore, the step-size parameters were defined by computer simulation, to reduce the system BER. In the experiments, the optimization of $\lambda$ (LMS) was performed assuming that the phase is perfectly estimated, and the optimization 
Table 2 Values of the steps of LMS $(\lambda)$ and PLL $(\rho)$ for different scenarios

\begin{tabular}{|c|c|c|c|c|}
\hline \multicolumn{3}{|c|}{ Scenario $1(\kappa=0.1, \mu=0.5)$} & \multirow{2}{*}{$\begin{array}{l}\mathbf{1 0 0} \mathbf{~ H z} \\
0.5\end{array}$} & \multirow{2}{*}{$\begin{array}{l}\mathbf{2 0 0} \mathbf{~ H z} \\
0.5\end{array}$} \\
\hline$M=4$ & $\theta=0.0^{\circ}$ & $\lambda$ & & \\
\hline & & $\rho$ & 0.5 & 0.5 \\
\hline & $\theta=29.2^{\circ}$ & $\lambda$ & 0.25 & 0.25 \\
\hline & & $\rho$ & 0.25 & 0.45 \\
\hline \multirow[t]{4}{*}{$M=16$} & $\theta=0.0^{\circ}$ & $\lambda$ & 0.35 & 0.5 \\
\hline & & $\rho$ & 0.3 & 0.5 \\
\hline & $\theta=10.7^{\circ}$ & $\lambda$ & 0.5 & 0.6 \\
\hline & & $\rho$ & 0.35 & 0.5 \\
\hline \multicolumn{3}{|c|}{ Scenario $2(\kappa=1.5, \mu=2.0)$} & $100 \mathrm{~Hz}$ & $200 \mathrm{~Hz}$ \\
\hline \multirow[t]{4}{*}{$M=4$} & $\theta=0.0^{\circ}$ & $\lambda$ & 0.85 & 0.5 \\
\hline & & $\rho$ & 0.3 & 0.8 \\
\hline & $\theta=41.0^{\circ}$ & $\lambda$ & 0.1 & 0.2 \\
\hline & & $\rho$ & 0.25 & 0.45 \\
\hline \multirow[t]{4}{*}{$M=16$} & $\theta=0.0^{\circ}$ & $\lambda$ & 0.1 & 0.2 \\
\hline & & $\rho$ & 0.7 & 0.8 \\
\hline & $\theta=35.1^{\circ}$ & $\lambda$ & 0.15 & 0.2 \\
\hline & & $\rho$ & 0.4 & 0.5 \\
\hline
\end{tabular}

Table 2 shows the optimized values of the parameters of the LMS and PLL algorithms $(\lambda$ and $\rho$ ). The step-size parameters were defined by computer simulation, to reduce the system BER. In the experiments, the optimization of $\lambda$ (LMS) was performed assuming that the phase is perfectly estimated, and the optimization of $\rho$ (PLL) considered the amplitude perfectly estimated.

of $\rho$ (PLL) considered the amplitude perfectly estimated. Table 2 shows the values obtained for the step parameters in different scenarios.

Based on the optimized values of $\lambda$ and $\rho$, Monte Carlo simulation was performed to evaluate the impact of channel estimation errors on the optimal rotation angle. Figure 15 presents the BER curves of the modulation diversity system as a function of the rotation angle, considering different parameters for the estimation algorithms (LMS and PLL), a 16-QAM system under a typical fading scenario $(\kappa=1.5, \mu=2.0)$, a sampling frequency of 270833 symbols/second, an SNR of $20 \mathrm{~dB}$ and $100 \mathrm{~Hz}$ of maximum Doppler frequency.

As can be seen in the figure, the presence of channel estimation errors modifies significantly the value of the optimum rotation angle, requiring that the optimization of $\theta$ considers the existence of errors in the estimation of channel amplitude and phase. However, to evaluate the efficiency of the modulation diversity when operating in the presence of estimation errors, in the following experiments, the same rotation angles obtained for perfectly estimated channels are used. Thus, the simulation results, considering the impact of channel estimation errors on the system BER (as a function of the channel SNR), are presented in Figure 16. The curves with rotation were generated using an interleaving depth of 1650 symbols for $f_{D}=100 \mathrm{~Hz}$ and 825 symbols for $f_{D}=200 \mathrm{~Hz}$. The performance curves with the absence of channel estimation errors were also included for comparison.

Although the estimation errors have modified the optimum value of $\theta$, the use of the modulation diversity technique have improved the performance of the QPSK system $\left(f_{D}=100 \mathrm{~Hz}\right)$ when compared to conventional systems (i.e., systems that do not use this technique, or $0.0^{\circ}$ ), as can be seen in Figure 16a. As can be seen in the figure, the rotated scheme outperforms the conventional system by approximately $3.65 \mathrm{~dB}$ for a BER value of $4.13 \times 10^{5}$.

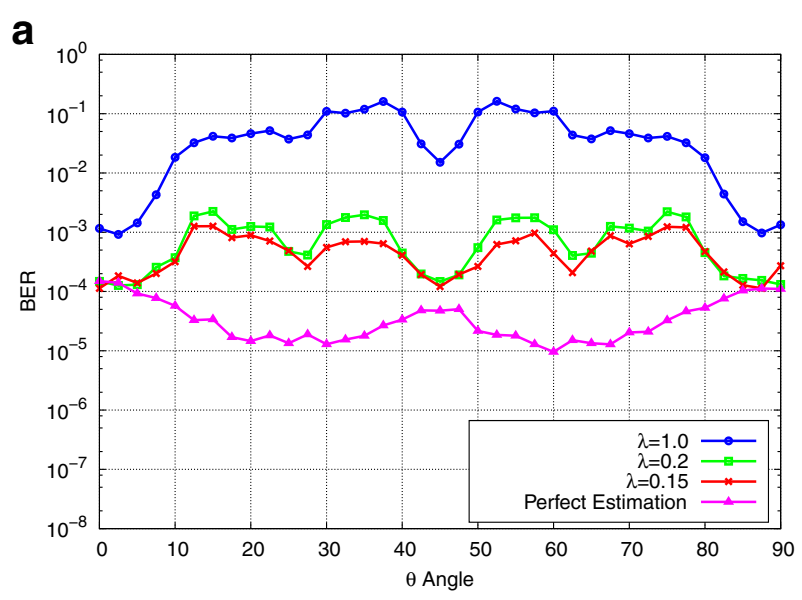

Amplitude Estimation Error (LMS algorithm used). b

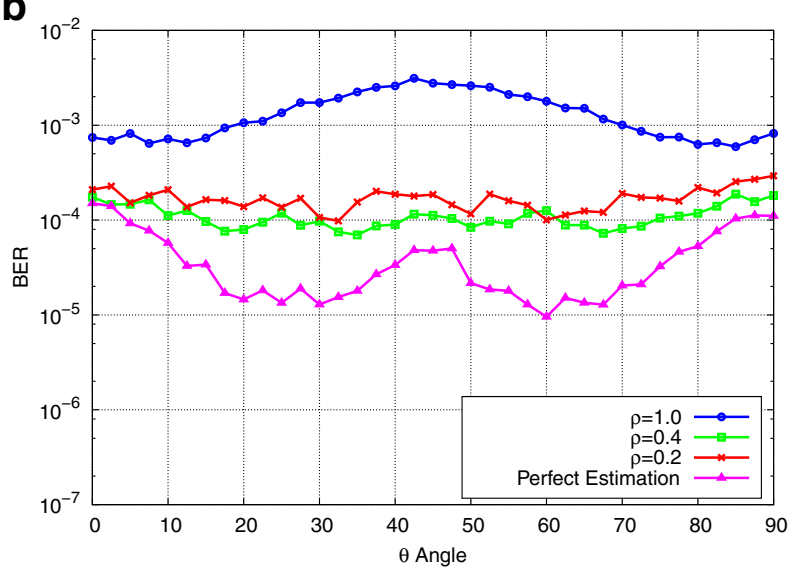

Phase Estimation Error (PLL algorithm used).

Figure 15 BER of the modulation diversity system, subject to amplitude and phase estimation errors, as a function of the rotation angle. The evaluation was performed considering the optimized values of $\lambda$ and $\rho$, a 16-QAM system under a typical fading scenario $(\kappa=1.5, \mu=2.0)$, a sampling frequency of 270833 symbols/second, an SNR of $20 \mathrm{~dB}$ and $100 \mathrm{~Hz}$ of maximum Doppler frequency. 

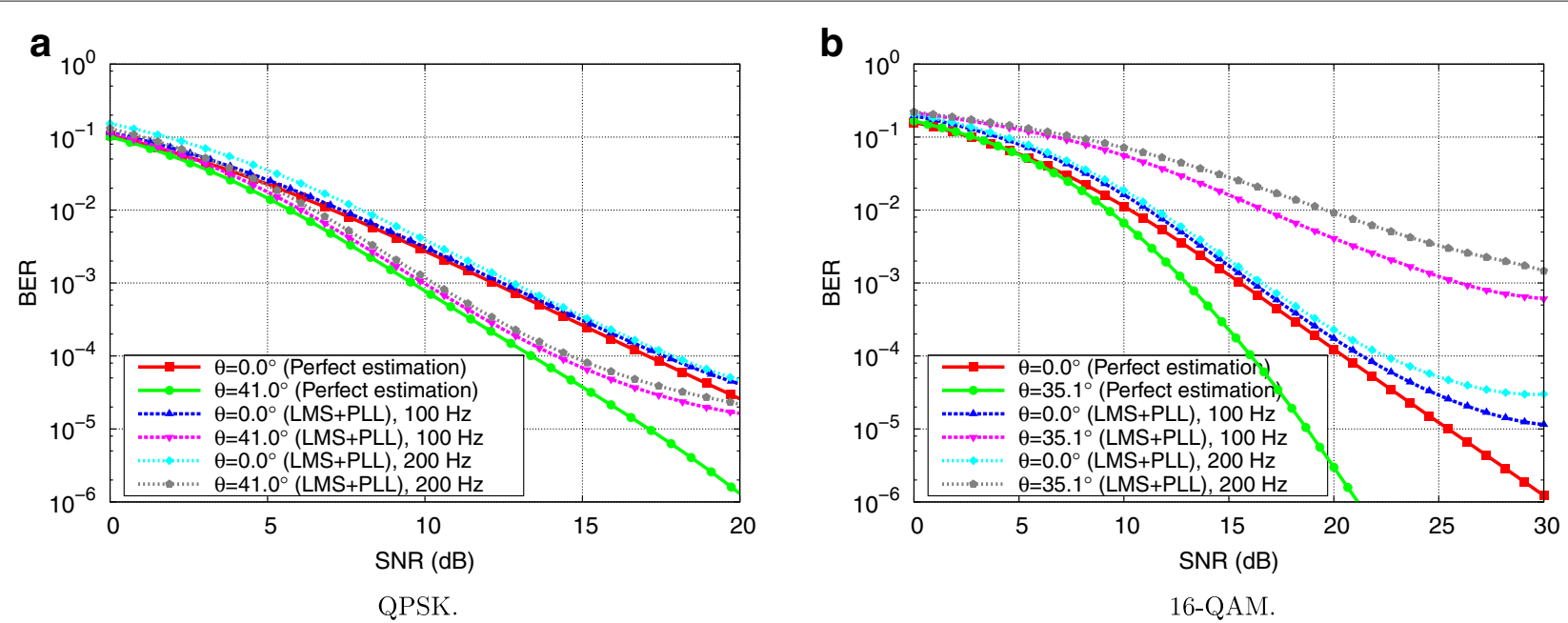

Figure 16 BER of the modulation diversity system, subject to amplitude and phase estimation errors, as a function of the channel SNR.

The evaluation was performed considering a QPSK and a 16-QAM system under a typical fading scenario $(\kappa=1.5, \mu=2.0)$, a sampling frequency of 270833 symbols/second and maximum Doppler frequencies of 100 and $200 \mathrm{~Hz}$.

However, the same conclusion cannot be obtained if a 16-QAM system is considered (Figure 16b). Instead, the use of a non-optimal rotation angle $\left(35.1^{\circ}\right)$ has significantly degraded the performance of the conventional system $\left(0.0^{\circ}\right)$. The degradation caused by the incorrect choice of the rotation angle can be confirmed comparing the BER for both $\theta$ values in Figure 15. For a BER of $6.3 \times 10^{-4}$, a loss of approximately $12.92 \mathrm{~dB}$ is observed when comparing the rotated and unrotated systems.

Finally, as a consequence of the presence of channel estimation errors in correlated fast fading channels, a bit error rate floor appear in the curves. The error floor increases with the value of the maximum Doppler frequency $\left(f_{D}\right)$. That happens because, for higher values of $f_{D}$, the channel variations are faster, increasing the number of estimation errors generated by LMS and PLL algorithms.

\section{Conclusions and future research}

The used fading models provide flexibility to characterize wireless channels in terms of measurable physical parameters. The recently proposed $\kappa-\mu$ model is a general fading distribution that can be used to represent the small-scale variation of the fading signal in a line-of-sight condition. The versatility obtained with the use of the $\kappa-\mu$ distribution provides a good fit to experimental data (particularly for low values of the fading envelope).

Diversity techniques are important resources to mitigate the effect of fading in wireless communications. Modulation diversity represents a relevant technique which combines a reference constellation rotation angle $\theta$ with the independent interleaving of the symbol components.

This article presented a performance evaluation of the modulation diversity technique for $\kappa-\mu$ fading channels.
To the best of authors' knowledge, the performance of the modulation diversity technique has not been evaluated considering the $\kappa-\mu$ fading model. Novel expressions to calculate the PEP of modulation diversity systems subject to $\kappa-\mu$ fading are derived, and they are numerically evaluated and compared. Finally, Monte Carlo simulations were used to evaluate the performance of the modulation diversity technique and to compare communication systems with and without this technique.

In actual systems, the performance of the modulation diversity can be affected by different impairments, such as the temporal correlation and the presence of estimation errors. Thus, different evaluations were performed in order to verify the impact of those impairments in the performance of the system.

When evaluating the modulation diversity in correlated channels, the authors have concluded that, if the interleaving depth is appropriately defined, the overall system performance is improved in correlated channels. The correct choice of the interleaving depth reduces the system BER to lower values than the obtained in uncorrelated channels (i.e., channels with $f_{D} \rightarrow \infty$ ). Furthermore, the use of the optimal interleaving depth does not cause significant changes in the optimum rotation angle.

On the other hand, the estimation errors modify significantly the optimum rotation angle value, requiring that the optimization of $\theta$ consider the presence of these errors in the system (in order to minimize the system BER). In some experiments (with QPSK systems) the performance loss caused by the estimation errors does not exceed the gain obtained by the modulation diversity technique, but this not occurs in all cases (e.g., in 16-QAM systems). 
Finally, the authors have verified that in the correlated channels, the estimation errors create a bit error rate floor, whose values increase with the value of the maximum Doppler frequency $\left(f_{D}\right)$.

Future research includes the evaluation of the modulation diversity technique in $\eta-\mu$ fading channels. The authors also intend to develop closed-form form expressions or more accurate approximations for the symbol error probability of those systems.

\section{Endnote}

${ }^{\text {a }}$ The value of the $F_{1}(\cdot)$ function in (23) is greater than or equal to 1 .

\section{Competing interests}

The authors declare that they have no competing interests.

\section{Acknowledgements}

The authors would like to thank COPELE/UFCG, Fapema, Federal Institute of Maranhão (IFMA), lecom and Capes for supporting the development of this research.

\section{Author details}

${ }^{1}$ D.Sc. Student of the Federal University of Campina Grande (UFCG), Electrical Engineering Post Graduate Program-COPELE, Campina Grande, Brazil. ${ }^{2}$ Department of Electrical Engineering, Federal University of Campina Grande, Campina Grande, Brazil. ${ }^{3}$ Institute for Advanced Studies in Communications, Campina Grande, Brazil. ${ }^{4}$ Federal Institute of Maranhão, São Luís, Brazil.

Received: 21 March 2012 Accepted: 8 January 2013

Published: 29 January 2013

\section{References}

1. VM DaSilva, ES Sousa, Fading-resistant modulation using several transmitter antennas. IEEE Trans. Commun. 45(10), 1236-1244 (1997)

2. GJ Foschini, MJ Gans, On limits of wireless communications in a fading environment when using multiple antennas. Wirel. Personal Commun. 6 311-335 (1998). http://dx.doi.org/10.1023/A:1008889222784

3. VTarokh, N Seshadri, AR Calderbank, Space-time codes for high data rate wireless communication: performance criterion and code construction. IEEE Trans. Inf. Theory. 44(2), 744-765 (1998)

4. WTA Lopes, F Madeiro, JF Galdino, MS Alencar, in Proceedings of the 64th IEEE Vehicular Technology Conference 2006 Fall (VTC'2006Fall), vol. 1. Impact of the estimation errors and Doppler effect on the modulation diversity technique, Montréal, Canada, 2006), pp. 1-5

5. SB Slimane, An improved PSK scheme for fading channels. IEEE Trans. Veh. Technol. 47(2), 703-710 (1998)

6. DP Andrez-Calderón, AC Oria, J García, P López, V Baena, I Lacadena, in XXIV Conference on Design of Circuits and Integrated Systems. Rotated constellation for DVB-T2, Zaragoza, Spain, 2009). http://dcis2009.unizar. es/index.php

7. G Taricco, E Viterbo, Performance of component interleaved signal sets for fading channels. IEE Electron. Lett. 32(13), 1170-1172 (1996)

8. MN Khormuji, UH Rizvi, GJM Janssen, SB Slimane, in 10th IEEE Singapore International Conference on Communication systems, vol. 1. Rotation optimization for MPSKMMQAM signal constellations over Rayleigh fading channels, Singapore, 2006), pp. 1-5

9. J Boutros, E Viterbo, Signal space diversity: a power- and bandwidth-efficient diversity technique for the Rayleigh fading channel. IEEE Trans. Inf. Theory. 44(4), 1453-1467 (1998)

10. J Kim, I Lee, in IEEE International Conference on Communications, vol. 1. Analysis of symbol error rates for signal space diversity in Rayleigh fading channels, Beijing, China, 2008), pp. 4621-4625

11. A Yilmaz, O Kucur, Performance of rotated PSK modulation in Nakagamim fading channels. Elsevier Digital Signal Process. 21(2), 296-306 (2011). http://www.sciencedirect.com/science/article/pii/S105120041000151X
12. NF Kiyani, JH Weber, AG Zajic, GL Stuber, in IEEE 68th Vehicular Technology Conference (VTC 2008-Fall), vol. 1. Performance analysis of a system using coordinate interleaving and constellation rotation in Rayleigh fading channels, Calgary, Canada, 2008), pp. 1-5

13. S Ozyurt, O Kucur, I Altunbas, Error performance of rotated phase shift keying modulation over fading channels. Wirel. Personal Commun. 43 1453-1463 (2007). http://dx.doi.org/10.1007/s11277-007-9319-7

14. J Kim, W Lee, J-K Kim, I Lee, On the symbol error rates for signal space diversity schemes over a Rician fading channel. IEEE Trans. Commun. 57(8), 2204-2209 (2009)

15. NF Kiyani, JH Weber, in IEEE Global Telecommunications Conference, vol. 1. Performance analysis of a partially coherent system using constellation rotation and coordinate interleaving, New Orleans, USA, 2008), pp. 1-5

16. S Stein, Fading channel issues in system engineering. IEEE J. Sel. Areas Commun. 5(2), 68-89 (1987)

17. MD Yacoub, The $\kappa-\mu$ distribution and the $\eta-\mu$ distribution. IEEE Antennas Propag. Mag. 49, 68-81 (2007)

18. KP Peppas, Sum of nonidentical squared $\kappa$ - $\mu$ variates and applications in the performance of analysis of diversity receivers. IEEE Trans. Veh. Technol. 61, 413-419 (2012)

19. X Wang, NC Beaulieu, Switching rates of two-branch selection diversity in $\kappa-\mu$ and $\alpha-\mu$ distributed fadings. IEEE Trans. Wirel. Commun. 8(4), 1667-1671 (2009)

20. SL Cotton, WG Scanlon, J Guy, The $\kappa-\mu$ distribution applied to the analysis of fading in body to body communication channels for fire and rescue personnel. IEEE Antennas Wirel. Propag. Lett. 7, 66-69 (2008)

21. SL Cotton, US Dias, WG Scanlon, MD Yacoub, On the distribution of signal phase in body area networks. IEEE Commun. Lett. 14(8), 728-730 (2010)

22. IS Gradshteyn, IM Ryzhik, Table of Integrals, Series, and Products, 6th edn. (Academic, New York, 2000)

23. JG Proakis, Digital Communications, 4th edn. (McGraw-Hill, New York, 2001)

24. JW Craig, in IEEE Military Communications Conference (MILCOM '91), vol. 2. A new, simple and exact result for calculating the probability of error for two-dimensional signal constellations, McLean, Virginia, USA, 1991), pp. $571-575$

25. D Sanders, FV Glehn, US Dias, in XXIX Brazilian Symposium on Telecommunications (SBrT 2011). Spectrum sensing over $\kappa-\mu$ fading channel, Curitiba, PR, 2011). http://www.sbrt.org.br/sbrt2011/

26. WH Press, SA Teukolsky, WT Vetterling, BP Flannery (eds.), Numerical Recipes: The Art of Scientific Computing, 3rd edn. (Cambridge University Press, New York, 2007)

27. TS Rappaport, Wireless Communications: Principles and Practice, 2nd edn. (Prentice Hall, Upper Saddle River, 1996)

28. WC Jakes (ed.), Microwave Mobile Communications (John Wiley \& Sons Inc., New York, 1975)

29. $\mathrm{RH}$ Clarke, A statistical theory of mobile radio reception. Bell Systs. Tech. J. 47(6), 957-1000 (1968)

30. MD Yacoub, Foundations of, Mobile Radio Engineering, 1st edn. (CRC Press, Inc., Boca Raton, FL, 1993)

31. MC Jeruchim, P Balaban, KS Shanmugan (eds.), Simulation of, Communication Systems: Modeling, Methodology and Techniques (Kluwer Academic Publishers, Norwell, MA, 2000)

32. S Haykin, Adaptive Filter Theory, 4th edn. (Prentice Hall, New Jersey, USA, 2002)

33. P Koufalas, State variable approach to carrier phase recovery and fine automatic gain control on flat fading channels, Master's thesis, School of Physics and Electronic Systems Engineering. University of South Australia (1996)

doi:10.1186/1687-1499-2013-17

Cite this article as: Lopes et al:: Performance of the modulation diversity technique for $\kappa-\mu$ fading channels. EURASIP Journal on Wireless Communications and Networking 2013 2013:17. 